\title{
A “TITS-ALTERNATIVE" FOR SUBGROUPS OF SURFACE MAPPING CLASS GROUPS
}

\author{
BY \\ JOHN MCCARTHY
}

\begin{abstract}
It has been observed that surface mapping class groups share various properties in common with the class of linear groups (e.g., [BLM, H]). In this paper, the known list of such properties is extended to the "Tits-Alternative", a property of linear groups established by J. Tits [T]. In fact, we establish that every subgroup of a surface mapping class group is either virtually abelian or contains a nonabelian free group.

In addition, in order to establish this result, we develop a theory of attractors and repellers for the action of surface mapping classes on Thurston's projective lamination spaces [Th1]. This theory generalizes results known for pseudo-Anosov mapping classes [FLP].
\end{abstract}

0. Introduction. Let $F$ be a complete, orientable Riemann surface of finite type, without holes, with $c$ connected components, $b$ cusps and genus $g$. We require that each connected component of $F$ has negative Euler characteristic. We assume that $F$ is equipped with a complete hyperbolic structure. $\partial F$ will denote a family of disjoint simple closed curves, each bounding a cusp of $F$. We shall assume that each component of $\partial F$ is a horocycle in the cusp.

The mapping class group of $F, \Gamma(F)$, is the group of isotopy classes of diffeomorphisms of $F$. The elements of this group are called mapping classes [B1, B2, Ha, HT]. The main result of this paper arose from a consideration of properties which $\Gamma(F)$ shares in common with the class of linear groups. Other examples of this theme occur in [BLM and H]. The particular property of linear groups which is under consideration here was established by $\mathbf{J}$. Tits.

THEOREM [T]. Let $G$ be a subgroup of $\operatorname{GL}(n, k)$, where $k$ is a field of characteristic zero. Either $G$ contains a solvable subgroup of finite index, or $G$ contains a nonabelian free group.

This theorem has become known as the "Tits-Alternative for $\operatorname{GL}(n, k)$ " [BL]. In [BL], it is conjectured that this alternative holds for a much wider class of groups to which $\Gamma(F)$ belongs. (It is not known whether $\Gamma(F)$ embeds in $\operatorname{GL}(n, k)$.) In this paper, we prove that a stronger alternative holds in $\Gamma(F)$.

THEOREM A. Let $G$ be a subgroup of $\Gamma(F)$. Either $G$ contains an abelian subgroup of finite index, or $G$ contains a nonabelian free group.

Received by the editors March 19, 1984 and, in revised form, October 17, 1984.

1980 Mathematics Subject Classification. Primary 57M99; Secondary 20F38.

Key words and phrases. Surface mapping class group, geodesic lamination, virtually abelian.

(C)1985 American Mathematical Society $0002-9939 / 85 \$ 1.00+\$ .25$ per page 
Note. This result has been proven simultaneously by N. Ivanov of the Leningrad Branch of the Steklov Mathematical Institute, using similar methods.

$G$ is virtually abelian if $G$ contains an abelian subgroup of finite index. (Similarly, one can define various virtual properties such as virtually solvable and virtually torsion free.) This stronger alternative for $\Gamma(F)$ was expected due to the following theorem in [BLM].

THEOREM [BLM]. Every solvable subgroup of $\Gamma(F)$ is virtually abelian.

If $S$ is a subset of $\Gamma(F)$, let $\operatorname{gp}(S)$ denote the subgroup of $\Gamma(F)$ which is generated by $S$. In the course of proving Theorem A, we will prove a more exact statement for two generator subgroups of $\Gamma(F)$.

TheOREM B. Let $\sigma$ and $\tau$ be elements of $\Gamma(F)$. There exist nonzero integers, $M$ and $N$, such that either $\operatorname{gp}\left(\sigma^{M}, \tau^{N}\right)$ is abelian, or $\operatorname{gp}\left(\sigma^{M}, \tau^{N}\right)$ is a nonabelian free group of rank two on the generators $\sigma^{M}$ and $\tau^{N}$.

The central idea behind the proof of Theorems A and B is a criterion for free groups which was employed by Tits in the proof of his theorem for $\operatorname{GL}(n, k)[\mathrm{T}]$.

FREEDOM CRITERION. Let $G$ be a group acting on a set $X, \sigma, \tau \in G, U, V \subset X$ and $p \in X \backslash(U \cup V)$. In addition, suppose that for all nonzero integers $n$,

(1) $\sigma^{n}(\{p\} \cup V) \subset U$ and

(2) $\tau^{n}(\{p\} \cup U) \subset V$.

Then $\operatorname{gp}(\sigma, \tau)$ is a free group of rank two on the generators $\sigma$ and $\tau$.

In order to put this criterion to work, we consider the action of $\Gamma(F)$ on $\mathscr{P} \mathscr{L}(F)$, the projective lamination space introduced by Thurston [Th1]. We associate to each mapping class $\tau$ a pair of subsets of $\mathscr{P} \mathscr{L}(F),\left(\operatorname{Join}_{+}(\tau)\right.$, Join $\left.\_(\tau)\right)$. We prove that these objects serve as attractors and repellers in the following sense.

Uniform Convergence Lemma. Let $\tau \in \Gamma(F)$, let $K$ be a compact subset of $\mathscr{P} \mathscr{L}(F) \backslash$ Join_ $(\tau)$, and let $U$ be an open neighborhood of $\operatorname{Join}_{+}(\tau)$. There exists a positive integer $N$ such that if $n \geqslant N$, then $\tau^{n}(K) \subset U$.

From Thurston's theory of surface mapping classes [Th2, FLP], we observe that each mapping class $\tau$ has a canonical reduction to a simple direct sum of a mapping class of algebraically finite type and a pseudo-Anosov mapping class. (Of course, we must allow that one of the "factors" is not present.) Although the dynamics of the actions of these two classes of maps on projective lamination spaces are distinct (the former being "parabolic" and the latter being "hyperbolic"), they nevertheless have the common property expressed by the Uniform Convergence Lemma.

For the first "factor", we construct a function which vanishes on Join ${ }_{+}(\tau)$ which is equal to Join_( $\tau)$. By observing that the Uniform Convergence Lemma is "invariant" under iteration of $\tau$, we are able to replace $\tau$ by any given iterate. Since, up to iteration, algebraically finite type mapping classes are "direct products" of Dehn twists, we are able, without loss of generality, to give explicit calculations of the action of $\tau$ on the projective lamination space in terms of natural coordinates supplied by the Harer-Penner theory of pavings [HP]. In this manner, we are able to 
establish that, under iteration of $\tau$, the given function converges to zero with the appropriate uniformity, and establish, thereby, the Uniform Convergence Lemma for mapping classes of algebraically finite type.

For the second "factor", the Uniform Convergence Lemma for pseudo-Anosovs is established by similar arguments involving the geometric intersection form of Thurston.

Subsequently, by employing the natural topological join structure on projective lamination spaces of surfaces of several components, we are able to "join" the "height" functions constructed for the previous two classes of maps in order to obtain "height" functions for the simple direct sums. By a more careful analysis of the convergence of the "composite" functions, we are able to deduce the Uniform Convergence Lemma for simple direct sums.

Finally, in order to compare the dynamics of an arbitrary mapping class with that of its corresponding reduction to a simple direct sum, we construct natural reduction maps between the corresponding projective lamination spaces. It is then a rather straightforward argument to deduce the general statement of the Uniform Convergence Lemma from the previous results.

Here is an outline of the paper. In $\S 1$, we review the basic aspects of Thurston's theory of geodesic laminations. In $\$ 2$, we review the outlines of Thurston's theory of surface mapping classes. The reader who is familiar with this material may wish to begin with $\$ 3$.

In the following two sections, $\$ \S 3$ and 4 , we establish the Uniform Convergence Lemma for mapping classes of algebraically finite type and the Uniform Convergence Lemma for pseudo-Anosovs. Combining these results, we deduce, in $\$ 5$, the Uniform Convergence Lemma for simple direct sums.

In $\S 6$, we employ the theory of pavings to construct the aforementioned reduction maps. We employ these maps, in $\S 7$, to give the general definition of the Joins of surface mapping classes. In the same manner in which these classes "decompose" into canonical "parts", the Joins "decompose" into canonical "parts". Although it is possible to give an "intrinsic" definition of the Joins [Mc2], the use of the reduction maps captures the "anatomy" directly. In $§ 8$, we employ the reduction maps and the description of the Joins to deduce the Uniform Convergence Lemma in full generality.

In $\S 9$, we prove Theorem $\mathrm{B}$ and in $\$ 10$, we prove Theorem $\mathrm{A}$. Both theorems follow fairly directly from the results of the previous sections.

1. Laminations and train tracks. The reader is referred to [Ke, M, Th1, Th3, Th4 and Th5] for general discussions of measured geodesic laminations, to [FLP and TH2] for expositions of the parallel theory of measured foliations, and finally to [HP] for a detailed discussion of the theory of measured train tracks and pavings. At this point, we shall review some of the definitions for the purposes of establishing terminology and notation.

A geodesic lamination on $F$ is a foliation of a closed subset of $F$ all of whose leaves are complete geodesics. The simplest examples of geodesic laminations are provided 
by families of disjoint simple closed geodesics. We shall refer to such a family as an admissible system $[\mathbf{G}]$.

A transverse measure on a geodesic lamination is a family of finite Borel measures supported on the local cross sections and compatible with the transition functions. An admissible system has a natural transverse measure. The local cross sections of an admissible system are finite sets which we can equip with the counting measure. The resulting transverse measure is referred to as the counting measure on the admissible system.

A measured lamination, $\mathscr{L}$, is a pair $(\gamma, \mu)$, where $\gamma$ is a geodesic lamination and $\mu$ is a transverse measure for $\gamma$. The support of $\mathscr{L}$ is the underlying lamination $\gamma$, which we shall also denote as $\operatorname{supp}(\mathscr{L})$. Unless otherwise specified, we shall consider an admissible system as a measured lamination with support, the admissible system, and transverse measure, the counting measure. $\mathscr{M} \mathscr{L}(F)$ is the set of all measured laminations.
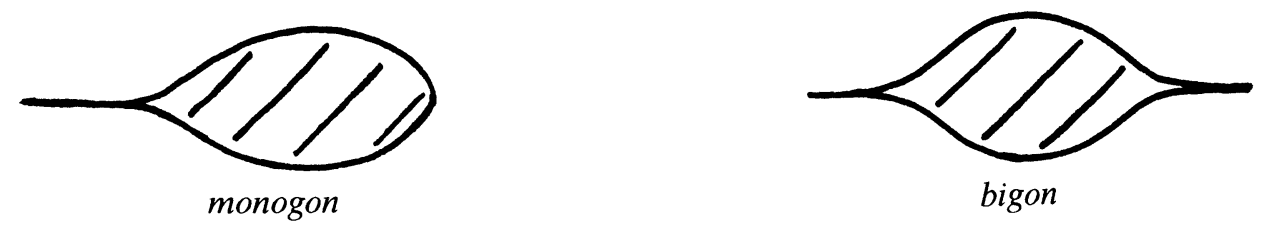

FiguRE 1

A train track $T$ on $F$ is a $C^{1}$-graph properly embedded in $F$ such that $F \backslash T$ has no monogons or bigons (Figure 1). A geodesic lamination $\gamma$ of $F$ is carried by $T$ if there is a proper $C^{1}$ homotopy $\psi: F \times I \rightarrow F$, such that

(1) $\psi_{0}=$ identity,

(2) $\psi_{1}(\gamma) \subset T$, and

(3) $\left.D \psi_{t}\right|_{\gamma}$ is never zero.

The map $\psi_{1}$ is called a collapsing map. We say that it collapses $\gamma$ to $T$.

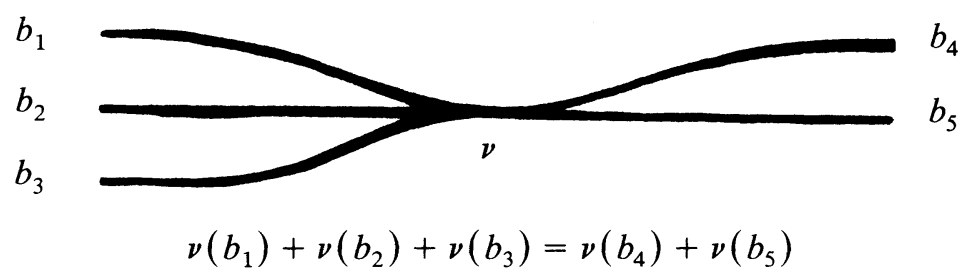

FIGURE 2

A transverse measure for $T$ is a nonnegative function $\nu$ on the unoriented branches of $T, \beta(T)$, such that at every vertex of $T$, the sum of the values of the branches on either side of the vertex are equal. A vertex of $T$ is called a switch and we say that $\nu$ satisfies the switch conditions (Figure 2). A measured train track is a pair $(T, \nu)$, where $T$ is a train track and $\nu$ is a transverse measure for $T$. 

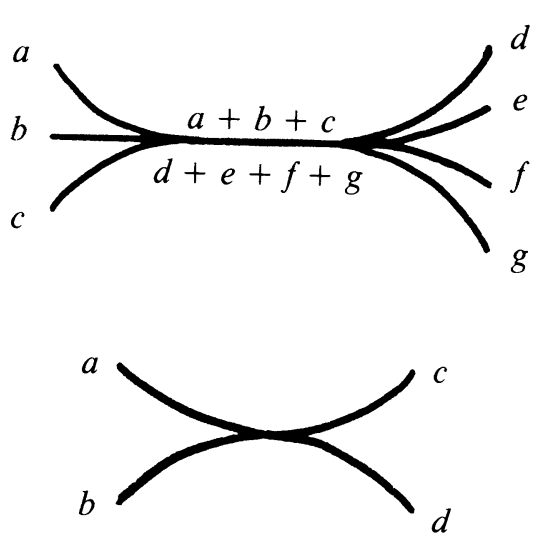

$g$

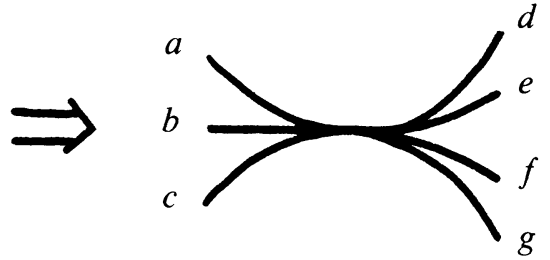

$g$

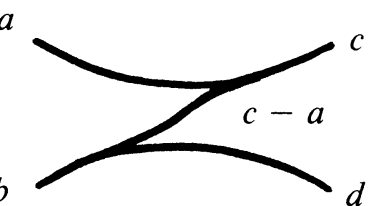

FIGURE 3

If $\mathscr{L}$ is a measured lamination $(\gamma, \mu)$, with $\gamma$ carried by $T$, we shall say $\mathscr{L}$ is carried by $T$. In such an event, we obtain a measured train track $\left(T, \mu_{\#}\right)$ associated to $\mathscr{L}$. The value $\mu_{\#}(b)$, where $b$ is a branch of $T$, may be regarded as "the total transverse measure of the local leaves whose image under the collapsing map runs through the given edge $b$ " [M].

Harer and Penner define an equivalence relation on measured train tracks which is generated by the following topological moves:

(1) isotopy of $T$,

(2) erasing branches with zero transverse measure,

(3) valid Whitehead moves (Whitehead moves respecting the switch conditions as in Figure 3).

Harer and Penner obtain a purely combinatorial definition of $\mathscr{M} \mathscr{L}(F)$ by establishing that the notion of a measured lamination being carried by a train track provides a one-to-one correspondence between the set of measured laminations and the set of equivalence classes of measured train tracks [HP].

Note. The additional technical restrictions on the train tracks required to insure this correspondence (i.e. the notions of recurrent and transversely recurrent), will not be significant for our purposes.

Let $\mathscr{L}$ be a measured lamination $(\gamma, \mu)$ on $F$ and let $\lambda$ be a positive real. We may multiply each of the local transverse measures of $\mu$ by the real $\lambda$ to obtain a new transverse measure which we denote as $\lambda \cdot \mu$. There is an action of $\mathbf{R}_{+}$on $\mathscr{M} \mathscr{L}(F)$ by the rule: $\lambda \cdot \mathscr{L}=(\gamma, \lambda \cdot \mu)$. If we let $\overline{\mathbf{R}_{+}}=\mathbf{R}_{+} \cup\{0\}$, we may extend this action, in an obvious fashion, to an action of $\overline{\mathbf{R}_{+}}$on $\mathscr{M} \mathscr{L}(F)$. The obvious action of $\overline{\mathbf{R}_{+}}$on the set of measured train tracks evidently descends to this action under the correspondence outlined above.

In addition to the action of $\overline{\mathbf{R}_{+}}$on $\mathscr{M} \mathscr{L}(F)$, Thurston defines an action of $\Gamma(F)$ [Th1]. From the discussion in [M], it is possible to describe this action as follows. Let $\tau$ be a mapping class and $(\gamma, \mu)$ a measured lamination. One may choose a representative $t$ of $\tau$ such that $t(\gamma)$ is a geodesic lamination. Furthermore, $t(\gamma)$ has a natural transverse measure $t_{*}(\mu)$ induced from $\mu$ via $t$. We define $\tau(\gamma, \mu)=$ $\left(t(\gamma), t_{*}(\mu)\right)$. This gives a well-defined action on $\mathscr{M} \mathscr{L}(F)$. 
In a similar manner, $\Gamma(F)$ acts on equivalence classes of measured train tracks. Let $\tau \in \Gamma(F)$ and let $[(T, \nu)]$ be an equivalence class of a measured train track $(T, \nu)$. Choose a diffeomorphism $t$ representing $\tau$. If we apply $t$ to $T$, we obtain a train track $t(T)$. In addition, $t$ induces a bijection from $\beta(T)$ to $\beta(t(T))$ which we may use to transfer the measure $\nu$ to a transverse measure $t_{*}(\nu)$ on $t(T)$. Let $\tau[(T, \nu)]=\left[\left(t(T), t_{*}(\nu)\right)\right]$. It is evident that this action is well defined, and agrees with the action of $\Gamma(F)$ on $\mathscr{M} \mathscr{L}(F)$.

The reader is referred to [Ke and $\mathbf{M}$ ] for descriptions of Thurston's topology on $\mathscr{M} \mathscr{L}(F)$, in the case where $F$ is a connected surface. Harer and Penner give a combinatorial description of this topology as follows [HP]: Let $M(T)$ be the set of measured train tracks with the same underlying track $T$, let $\beta(T)$ be the set of unoriented branches of $T$ and let $\mathbf{R}^{\beta(T)}$ be the vector space of real valued functions on $\beta(T) . M(T)$ is an additive submonoid of this vector space invariant under the action of $\mathbf{R}_{+}$. Since $M(T)$ is given by a family of homogeneous linear equations (the switch conditions), and inequalities (nonnegativity), $M(T)$ is a cone over a polyhedron. (We give $M(T)$ the subspace topology, where $\mathbf{R}^{\beta(T)}$ is given the Euclidean topology.)

Assuming the train track may be isotoped in $F$ to a train track of small curvature, the Euclidean topology above is compatible with Thurston's topology. This is made precise by the following theorem.

THEOREM $1.1[\mathbf{M}]$. There exists a constant $K>0$, such that for any complete hyperbolic surface $F$ and any train track $T$ on $F$ of curvature less than $K$, if $U(T)$ is the subspace of $\mathscr{M} \mathscr{L}(F)$ consisting of measured laminations carried by $T$, then the map

$$
U(T) \rightarrow M(T), \quad(\gamma, \mu) \rightarrow\left(T, \mu_{\#}\right)
$$

is a homeomorphism.

Note. If $T^{\prime}$ is isotopic to $T$, then any isotopy between $T^{\prime}$ and $T$ induces a homeomorphism between $M\left(T^{\prime}\right)$ and $M(T)$. In addition, in this situation, it is evident that $U\left(T^{\prime}\right)=U(T)$. Hence, the theorem remains valid provided $T$ is isotopic to a train track of curvature less than $K$. (The notions of recurrent and transversely recurrent are the combinatorial conditions required to insure that a train track may be isotoped to one of sufficiently small curvature [HP].) Henceforth we assume that all train tracks are isotopic to train tracks of curvature less than $K$.

Harer and Penner show that, under the correspondences, $\mathscr{M} \mathscr{L}(F)$ is homeomorphic to the quotient space of the disjoint union of the cones defined above [HP].

The projective lamination space $\mathscr{P} \mathscr{L}(F)$ is simply the orbit space of $\mathscr{M} \mathscr{L}(F) \backslash\{0\}$ by the action of $\mathbf{R}_{+}$. We shall denote the projective class of a measured lamination $\mathscr{L}$ by $[\mathscr{L}]$. It is easy to see that the action of $\Gamma(F)$ on $\mathscr{M} \mathscr{L}(F)$ descends to an action on $\mathscr{P} \mathscr{L}(F)$. As pointed out in $[\mathbf{K e}], \mathscr{P}_{\mathscr{L}}(F)$ is given the quotient topology.

The reader will observe that the obvious action of $\mathbf{R}_{+}$on $M(T)$ is compatible with the defined action of $\mathbf{R}_{+}$on $\mathscr{M} \mathscr{L}(F)$. Hence, we have a projective version of Theorem 1.1 as well.

For our purposes, we shall require the following results. 
THEOREM 1.2 [Th1, HP]. Let $F$ be a connected orientable surface of genus $g$.

(1) (case a: $\partial F=\varnothing) \mathscr{P} \mathscr{L}(F) \cong S^{6 g^{-6-1}}$ (sphere),

(case b: $\mathrm{b} F \neq \varnothing) \mathscr{P} \mathscr{L}(F) \cong D^{6 g+2 b-6-1}$ (disc).

(2) The action $\overline{\mathbf{R}_{+}} \times \mathscr{M} \mathscr{L}(F) \rightarrow \mathscr{M} \mathscr{L}(F)$ is continuous.

(3) The action $\Gamma(F) \times \mathscr{M} \mathscr{L}(F) \rightarrow \mathscr{M} \mathscr{L}(F)$ is continuous.

(4) The action $\Gamma(F) \times \mathscr{P} \mathscr{L}(F) \rightarrow \mathscr{P} \mathscr{L}(F)$ is continuous.

Suppose that $F=\amalg_{i \in I} F_{i}$, where each $F_{i}$ is connected. Given any measured lamination $\mathscr{L}$ on $F$, we have associated measured laminations, given by restriction to the components. Therefore, we have a bijective correspondence between the two spaces $\mathscr{M} \mathscr{L}(F) \leftrightarrow \prod_{i \in I} \mathscr{M} \mathscr{L}\left(F_{i}\right)$. Giving the right-hand term the product topology, each factor being equipped with Thurston's topology, we use this bijection to induce a topology on $\mathscr{M} \mathscr{L}(F)$. As always $\mathscr{P} \mathscr{L}(F)$ is given the quotient topology.

As we shall demonstrate, the structure of $\mathscr{P} \mathscr{L}(F)$ for surfaces of several components may be understood in terms of the notion of topological join. At this point, we recall the definition of the join of topological spaces.

If $\left\{X_{i} \mid i \in I\right\}$ is a collection of topological spaces, then we may define the join of $\left\{X_{i} \mid i \in I\right\},{ }_{i \in I}^{*} X_{i}$, as the collection of formal sums

$$
\sum_{i \in I} \lambda_{i} \cdot x_{i}
$$

where $x_{i} \in X_{i}, \lambda_{i} \geqslant 0$ and $\sum_{i \in I} \lambda_{i}=1$. Equality of these formal sums is defined in an obvious manner. Likewise, the topology of the join is defined in an obvious fashion.

A weight function on $\mathscr{M} \mathscr{L}(F)$ is just a continuous function $\mid$ : $\mathscr{M} \mathscr{L}(F) \rightarrow \overline{\mathbf{R}_{+}}$ such that:

(1) $|\lambda \cdot \mathscr{L}|=\lambda \cdot|\mathscr{L}|$ for all $\lambda \in \overline{\mathbf{R}_{+}}, \mathscr{L} \in \mathscr{M} \mathscr{L}(F)$, and

(2) $|\mathscr{L}|=0$ if and only if $\mathscr{L}=0$.

For now, we assume such functions exist. Later, we shall have opportunity to construct explicit weight functions (\$3).

Now, suppose that $F=\amalg_{i \in I} F_{i}$. (We need not assume, in the following discussion, that any $F_{i}$ is connected.) We consider a fixed weight function | |.

LEMMA 1.3. $\mathscr{P} \mathscr{L}(F)$ is homeomorphic to $*_{i \in I} \mathscr{P} \mathscr{L}\left(F_{i}\right)$.

Proof. By the discussion above, $\mathscr{M} \mathscr{L}(F) \cong \prod_{i \in I} \mathscr{M} \mathscr{L}\left(F_{i}\right)$. It is easy to check that the following function yields the desired homeomorphism:

$$
\begin{aligned}
& \mathscr{P} \mathscr{L}(F) \rightarrow \underset{i \in I}{*} \mathscr{P} \mathscr{L}\left(F_{i}\right), \\
& {\left[\left(\mathscr{L}_{i}\right)_{i \in I}\right] \mapsto \sum_{i \in I}\left(\left|\mathscr{L}_{i}\right| / \mid \mathscr{L}_{\mid}\right) \cdot\left[\mathscr{L}_{i}\right]}
\end{aligned}
$$

REMARK. The homeomorphism of Lemma 1.3 is not natural. Unfortunately, there is no natural weight function. Nevertheless, we will find this structure to be very helpful.

Since the genus of a surface is just the sum of the genera of its components, it is easy to verify, using Lemma 1.3 and standard facts about topological joins, the following corollary of Theorem 1.2. 
COROLlaRY 1.4. Let $F$ be an orientable surface.

(1) (case a: $\partial F=\varnothing) \mathscr{P} \mathscr{L}(F) \cong S^{6 g-6 c-1}$ (sphere), (case b: $\mathrm{b} F \neq \varnothing) \mathscr{P} \mathscr{L}(F) \cong D^{6 g+2 b-6 c-1}$ (disc).

(2) The action $\overline{\mathbf{R}_{+}} \times \mathscr{M} \mathscr{L}(F) \rightarrow \mathscr{M} \mathscr{L}(F)$ is continuous.

(3) The action $\Gamma(F) \times \mathscr{M} \mathscr{L}(F) \rightarrow \mathscr{M} \mathscr{L}(F)$ is continuous.

(4) The action $\Gamma(F) \times \mathscr{P} \mathscr{L}(F) \rightarrow \mathscr{P} \mathscr{L}(F)$ is continuous.

2. Thurston's classification of mapping classes. The reader is referred to [BLM] for a discussion of notions related to Thurston's trichotomy for mapping classes. We shall give a brief review of the main features. First, we consider the case where $F$ is connected. A mapping class $\tau$ is pseudo-Anosov if there exists a unique pair [ $\left.\mathscr{L}_{-}\right]$and $\left[\mathscr{L}_{+}\right]$of projective classes of measured laminations preserved by the action of $\tau$ on $\mathscr{P} \mathscr{L}(F)$ [Th5]. In the event that $\tau$ is pseudo-Anosov, there exists a positive real, $\lambda>1$, such that $\tau \mathscr{L}_{ \pm}=\lambda^{ \pm 1} \mathscr{L}_{ \pm} ; \lambda$ is the dilatation factor for $\tau$. [ $\left.\mathscr{L}_{+}\right]$is the attracting lamination for $\tau$ and $\left[\mathscr{L}_{-}\right]$is the repelling lamination for $\tau$.

Now, suppose $F$ is an arbitrary surface. For any mapping class $\tau$ we may choose a nonzero exponent $n$, such that $\tau^{n}$ preserves each component of $F$. We refer to the restrictions of $\tau^{n}$ to these components as restrictions of $\tau$. The mapping class $\tau$ is pseudo-Anosov if each of its restrictions is pseudo-Anosov. The mapping class is of finite order if $\tau^{n}=1$ for some $n \neq 0$. The mapping class is reducible if it preserves some admissible system. We refer to such a system as a reduction system for $\tau$. Each isotopy class in the admissible system is a reduction class for $\tau$. If $\tau$ is reducible and $\mathscr{A}$ is a reduction system for $\tau$, we may choose a representative $t$ of $\tau$, such that $t(\mathscr{A})=\mathscr{A}$. Then $\left.t\right|_{F \backslash \mathscr{A}}$ determines a mapping class $\hat{\tau}$ in $\Gamma(F \backslash \mathscr{A})$, which we refer to as the reduction of $\tau$ along $\mathscr{A}$. We shall denote $F \backslash \mathscr{A}$ by $\hat{F}$.

Note. $\hat{F}$ is considered as a complete hyperbolic surface. Thus the structure on $\hat{F}$ is not that inherited directly from $F$. Being a surface of finite type and negative Euler characteristic, $\hat{F}$ does in fact have a complete hyperbolic structure. Although not unique, the choice of hyperbolic structure is not significant for our purposes. All the notions associated to laminations are known to be independent of the choice of hyperbolic structure; they are actually topological invariants of the underlying topological surface [Ke, M, Th5].

A mapping class $\tau$ is adequately reduced if each of its restrictions is either of finite order or pseudo-Anosov. A reduction system $\mathscr{A}$ for $\tau$ is an adequate reduction system for $\tau$ if the reduction of $\tau$ along $\mathscr{A}$ is adequately reduced.

The central result of Thurston's concerning mapping classes is as follows.

THEOREM 2.1 [Th3]. Every mapping class $\tau \in \Gamma(F)$ is either adequately reduced or has an adequate reduction system.

It appears to be convenient to speak of the empty set as an adequate reduction system for an adequately reduced mapping class. With this in mind, we may state the results of $[\mathbf{B L M}]$ concerning adequate reduction systems.

TheOREM 2.2 [BLM]. Every mapping class $\tau \in \Gamma(F)$ possesses a unique minimal adequate reduction system $\mathscr{A}_{\tau}$. 
The essential reduction system for $\tau$ is the canonical system of Theorem 2.2. The essential reduction of $\tau$ is the reduction of $\tau$ along $\mathscr{A}_{\tau}$.

3. The Uniform Convergence Lemma for mapping classes of algebraically finite type. A mapping class $\tau$ is of algebraically finite type if the essential reduction of $\tau$ is of finite order [N]. In this section, we shall prove the Uniform Convergence Lemma (U.C.L.) for mapping classes of algebraically finite type. Henceforth, throughout this section, $\tau$ denotes a fixed mapping class of algebraically finite type on a fixed surface $F$. It is easy to see that there exists a positive integer $M$ such that $\tau^{M}$ is a product of Dehn twists about the components of $\mathscr{A}_{\tau}[$ BLM].

In order to avoid the distraction of presenting a general definition of the Joins of mapping classes, we shall now give a special definition for this class of maps. (The reader may wish to turn to $\S 7$ for the general definition.) In this particular case, $\operatorname{Join}_{+}(\tau)=$ Join $_{-}(\tau)=$ the set of projective laminations whose supports have trivial transverse intersection with $\mathscr{A}_{\tau}$.

LEMMa 3.1. The Uniform Convergence Lemma is true for $\tau$ if and only if it is true for $\tau^{M}$.

Proof. By the properties of essential reduction systems, $\mathscr{A}_{\tau^{M}}=\mathscr{A}_{\tau}[\mathbf{B L M}]$. Clearly, therefore, $\tau^{M}$ is of algebraically finite type. Consequently, $\operatorname{Join}_{ \pm}\left(\tau^{M}\right)=\operatorname{Join}_{ \pm}(\tau)$.

It is evident, therefore, that the U.C.L. for $\tau$ implies the U.C.L. for $\tau^{M}$. On the other hand, suppose the U.C.L. is true for $\tau^{M}$. In order to establish it for $\tau$, let $K$ and $U$ be subsets of $\mathscr{P} \mathscr{L}(F)$ as in the hypotheses of the U.C.L. Let $U^{\prime}=\bigcap_{j=0}^{M-1} \tau^{-j}(U)$. Choose $N^{\prime}$ such that if $n>N^{\prime}$, then $\left(\tau^{M}\right)^{n}(K) \subset U^{\prime}$. Now let $N=M N^{\prime}$ and suppose that $n>N$. Then $n=q M+r$, where $q \geqslant N^{\prime}$ and $0 \leqslant r<M$. We conclude that $\tau^{n}(K)=\tau^{r}\left(\tau^{q M}(K)\right) \subset \tau^{r}\left(U^{\prime}\right)$. But $\tau^{r}\left(U^{\prime}\right) \subset \tau^{r}\left(\tau^{-r}(U)\right)=U$. Hence, $\tau^{n}(K) \subset$ $U$.

Henceforth, we may assume that $M=1$. With this assumption in mind, we shall begin with an explicit calculation of the action of $\tau$ on $\mathscr{M} \mathscr{L}(F)$ in terms of the coordinates of a paving. This amounts, in principle, to a calculation of the action of $\tau$ on the well-known Dehn-Thurston parameters for simple closed curves. This calculation has been carried out, in general, by Penner $[\mathbf{P}]$. Nevertheless, since our setting is much simpler, we shall give an explicit calculation here.

Using measured train tracks, Harer and Penner define their pavings of $\mathscr{P} \mathscr{L}(F)$ [HP]. Roughly speaking, a paving is a covering of $\mathscr{P} \mathscr{L}(F)$ by a finite family of polyhedra, any two of which meet along a common subface (not necessarily a codimension one subface), or are disjoint. In what follows, we allow these intersections to consist of a union of subfaces. We might call these "pseudo-pavings". They will suffice for our purposes. In fact, for our purposes, it will be easier to work with them. We shall call these "pseudo-pavings" simply pavings. We now review certain features which we shall need.

The first observation is simply that the projectivization of $M(T)$ is a polyhedron. Indeed, certain "maximal" train tracks correspond to polyhedra of maximal dimension in the manifold $\mathscr{P} \mathscr{L}(F)$. This idea is behind the Harer-Penner pavings. 

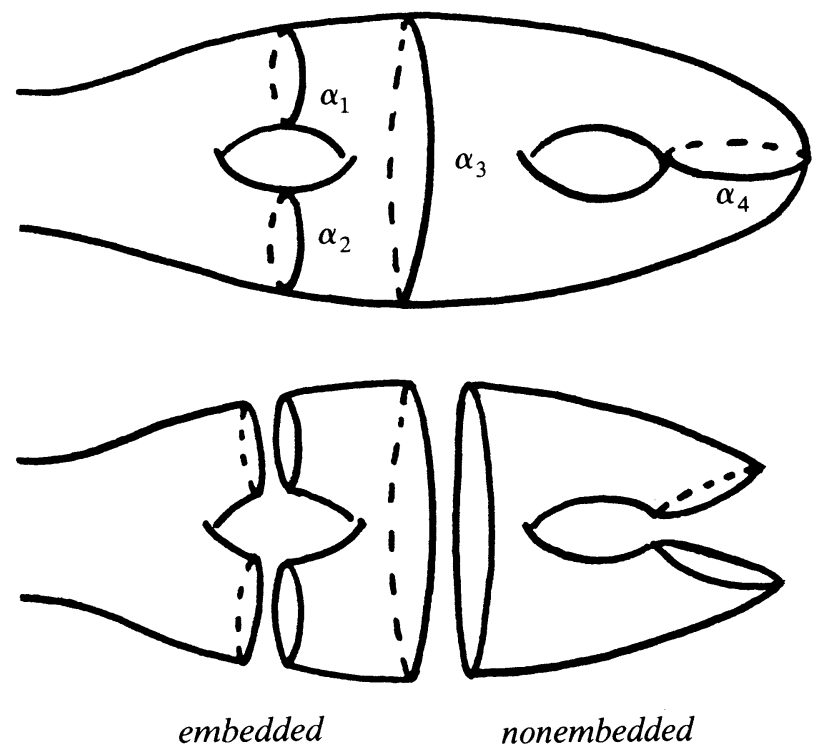

FIGURE 4

To construct a paving, one begins with a pants decomposition. A pants decomposition of $F$ is an admissible system $\mathscr{P}$ such that $F \backslash \mathscr{P}$ is a disjoint union of pairs of pants. (A pair of pants is a sphere with three holes.) For example, the collection $\left\{\alpha_{1}, \alpha_{2}, \alpha_{3}, \alpha_{4}\right\}$ of Figure 4 is a pants decomposition of the once-punctured surface of genus two. We do not require that the closed pants embed in $F$ (Figure 4).

About each component $\alpha$ of $\mathscr{P}$ we choose a closed annular neighborhood $A_{\alpha}$. In each cusp, we choose a horocycle $\beta$ and a punctured disc $D_{\beta}$ such that $\partial D_{\beta}=\beta$. These annuli and punctured discs are chosen to be pairwise disjoint. The complement of these annuli and punctured discs is a disjoint union of compact, embedded pairs of pants. We proceed to describe certain local models for a collection of train tracks.
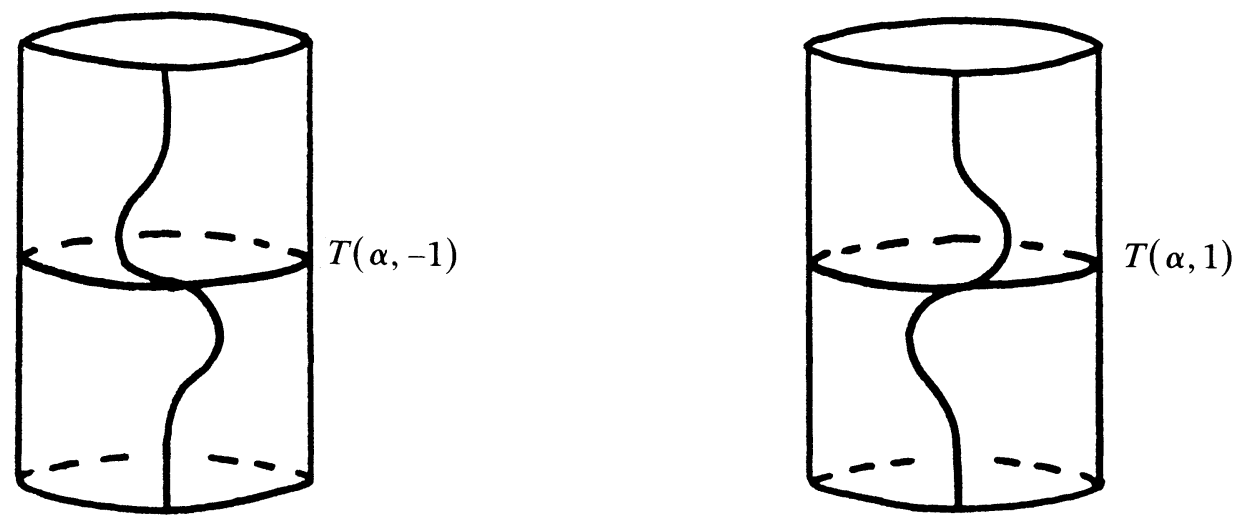

FIGURE 5 


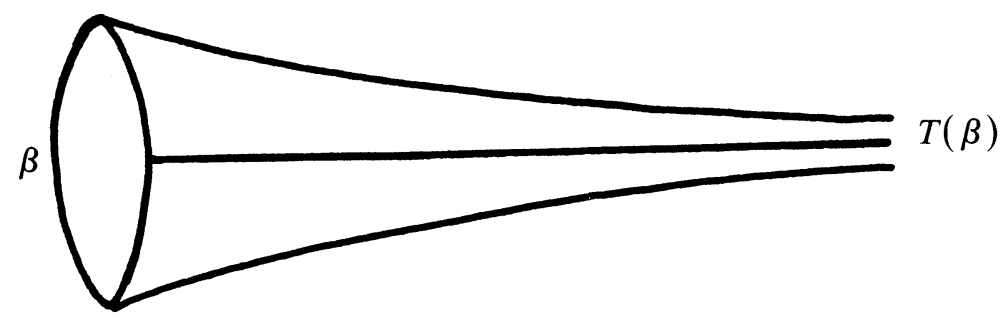

Figure 6

In each annulus $A_{\alpha}$, we choose two train tracks as depicted in Figure 5. In each cusp $D_{\beta}$ choose a train track such as in Figure 6.

In each compact, embedded pair of pants $P$, choose four train tracks as depicted in Figure 7. We assume that each of these local models is orthogonal to the corresponding boundary components. Furthermore, we require that they coincide on common boundary components. With these constraints, we may construct various train tracks by varying the choice of local model in each pair of pants and each annulus.

By fixing an ordering of the branches on each track $T$, we obtain, by Theorem 1.1, an embedding $j(T): U(T) \rightarrow \mathbf{R}^{B}$, where $B$ is the number of unoriented branches of $T$. We shall refer to such a map as a coordinate map on $U(T)$. In these coordinates, $U(T)$ is a cone over a polyhedron. (It is easy to verify that $B$ does not depend on the track $T$. In fact, $B=15 g+7 b-15 c$.)
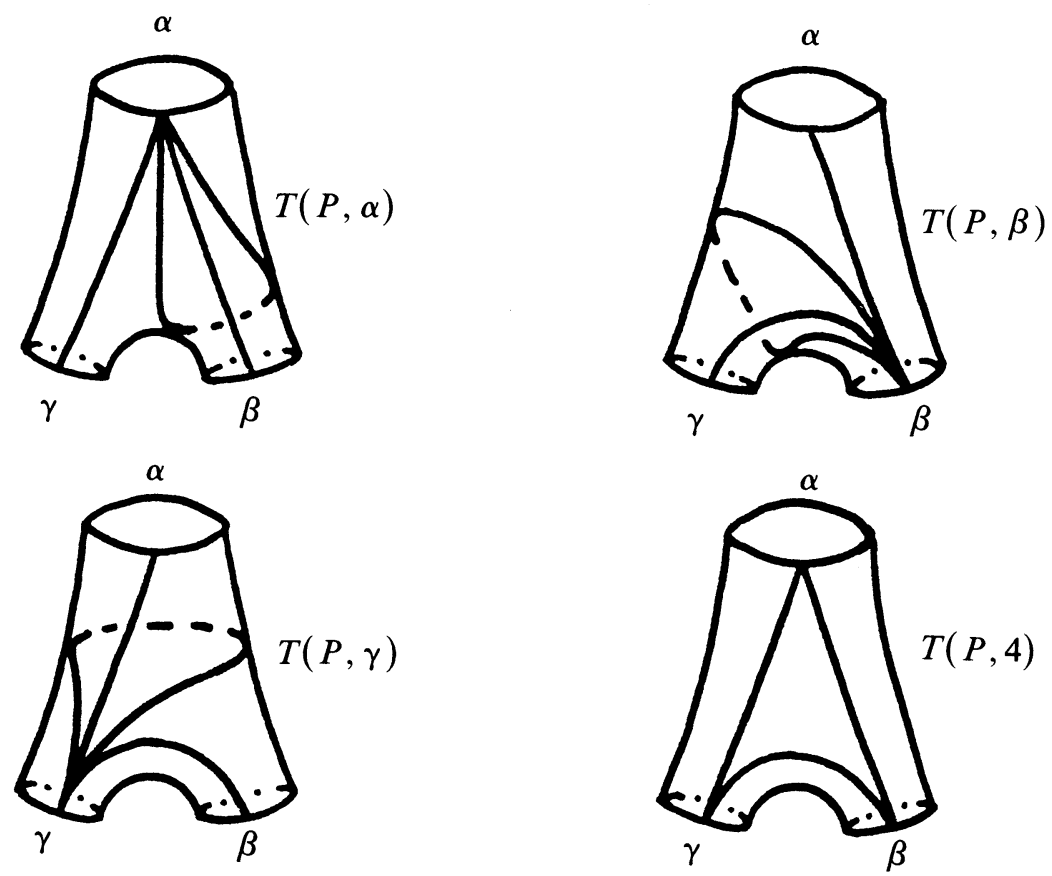

FIGURE 7 
In order to describe the transition functions for these coordinate maps we need to discuss the notion of subtrack. The coordinate maps have values in $\left(\overline{\mathbf{R}_{+}}\right)^{B}$. A face of $\left(\overline{\mathbf{R}_{+}}\right)^{B}$ is a subset of $\left(\overline{\mathbf{R}_{+}}\right)^{B}$ which is determined by a subset $J \subset I$, where $I=$ $\{1, \ldots, B\}$, and the conditions $x_{i}=0$ for each $i \in I \backslash J$. We shall denote such a face as $\left(\overline{\mathbf{R}_{+}}\right)^{J}$. It is, of course, naturally identified with $\left(\overline{\mathbf{R}_{+}}\right)^{A}$, where $A$ is the cardinality of $J$. Given such a face, $j(T)^{-1}\left(\left(\overline{\mathbf{R}_{+}}\right)^{J}\right)$ can be identified as $U(T(J))$, where $T(J)$ is the train track obtained from $T$ by "erasing" the edges corresponding to $I \backslash J$. We refer to $T(J)$ as a subtrack of $T$, and to $U(T(J))$ as a face of $U(T)$. If $T(J)$ is isotopic to $T^{\prime}\left(J^{\prime}\right)$, then it is easy to see that $U(T(J))=U\left(T^{\prime}\left(J^{\prime}\right)\right)$. Indeed, under the isotopy, the edges of $T$ corresponding to $J$ may be identified with the edges of $T^{\prime}$ corresponding to $J^{\prime}$. This yields a bijection $\sigma: J \rightarrow J^{\prime}$ which we arbitrarily extend to a bijection $\sigma: I \rightarrow I$. Evidently, if $\bar{\sigma}: \mathbf{R}^{B} \rightarrow \mathbf{R}^{B}$ is the isomorphism of $\mathbf{R}^{B}$ obtained by permuting coordinates according to $\sigma$, then

$$
\left.\overline{\boldsymbol{\sigma}} \circ j\left(T^{\prime}\right)\right|_{U\left(T^{\prime}\left(J^{\prime}\right)\right)}=\left.j(T)\right|_{U(T(J))} .
$$

Thus, the transition functions are just permutations of coordinates. We shall, henceforth, identify isotopic subtracks as above and refer to $T(J)$ as a common subtrack of $T$ and $T^{\prime}$. Likewise, we speak of $U(T(J))$ as a common face of $U(T)$ and $U\left(T^{\prime}\right)$.

Let $\mathscr{T}(\mathscr{P})$ be the collection of train tracks constructed from the local models chosen above. We are now ready to state the result from [HP] which we shall require.

THEOREM 3.2 [HP]. (1) $\mathscr{M} \mathscr{L}(F)=\bigcup\{U(T) \mid T \in \mathscr{T}(\mathscr{P})\}$.

(2) For each pair of cones $U(T)$ and $U\left(T^{\prime}\right), U(T) \cap U\left(T^{\prime}\right)$ is a finite union of common faces and the transition functions for the coordinate maps on these common faces are given by permutations of coordinates.

Note. The isotopies between common subtracks can be taken to fix $\mathscr{P}$.

The coordinate maps give homogeneous coordinates on polyhedra in $\mathscr{P} \mathscr{L}(F)$, and one obtains a covering of $\mathscr{P} \mathscr{L}(F)$ by polyhedra meeting along at most finitely many faces.

If $[\mathscr{L}]$ is a projective lamination with a representative $\mathscr{L}$ carried by a train track $T$, we say that $[\mathscr{L}]$ is carried by $T$. It is clear that if $[\mathscr{L}]$ is carried by $T$, any representative of $[\mathscr{L}]$ is carried by $T$. Let $\Delta(T)$ be the set of projective classes carried by $T$. The collection $\{\Delta(T) \mid T \in \mathscr{T}(\mathscr{P})\}$, with all the associated structure as a covering of $\mathscr{P} \mathscr{L}(F)$ by polyhedra with homogeneous coordinates, is called a paving of $\mathscr{P} \mathscr{L}(F)$ associated to the pants decomposition $\mathscr{P}$. We shall sometimes refer to the collection of cones $\{U(T) \mid T \in \mathscr{T}(\mathscr{P})\}$ as a paving for $\mathscr{M} \mathscr{L}(F)$.

REMARK. Harer and Penner use slightly different train tracks and obtain a covering by cones intersecting in at most one face. In addition they use only those polyhedra of maximal dimension. There is a simple combinatorial condition for determining which tracks yield cones of maximal dimension [HP]. We shall not concern ourselves with these refinements.

Suppose that $\mathscr{L} \in U(T)$. Let $j_{(T)}: U(T) \rightarrow \mathbf{R}^{B}$ be the coordinate map for $U(T)$. We may define the weight of $\mathscr{L},|\mathscr{L}|$, to be the sum of the coordinates. By Theorem 
$3.2,|\mathscr{L}|$ does not depend upon the choice of $T$ carrying $\mathscr{L}$. Therefore, we have a well-defined function

$$
\mid: \mathscr{M} \mathscr{L}(F) \rightarrow \overline{\mathbf{R}_{+}} .
$$

LEMMA 3.3.| $\mid: \mathscr{M} \mathscr{L}(F) \rightarrow \overline{\mathbf{R}_{+}}$is a weight function.

Proof. | | is continuous on each cone $U(T)$ and it is well defined on the intersections of these cones. Since these cones are closed subsets of $\mathscr{M} \mathscr{L}(F)$ and they cover $\mathscr{M} \mathscr{L}(F), \|$ is a continuous function.

It is easy to see that the given action of $\overline{\mathbf{R}_{+}}$on $U(T)$ agrees, under the identification given by the coordinate map $j(T)$, with the usual action of $\overline{\mathbf{R}_{+}}$on $\mathbf{R}^{B}$. Hence it is clear from the definitions that $|\lambda \cdot \mathscr{L}|=\lambda \cdot|\mathscr{L}|$ for all $\lambda \in \overline{\mathbf{R}_{+}}$and all $\mathscr{L} \in \mathscr{M} \mathscr{L}(F)$. Finally, it is clear that $|\mathscr{L}|=0$ if and only if $\mathscr{L}=0$.

Let $\mathscr{A}$ be the essential reduction system for $\tau$. Choose a pants decomposition $\mathscr{P}$ of $F$ such that $\mathscr{A} \subset \mathscr{P}$. We say that the paving of $\mathscr{P} \mathscr{L}(F)$ associated to $\mathscr{P}$ is adapted to $\mathscr{A}$. We may write $\tau=\prod_{\alpha \in \mathscr{A}}\left(\tau_{\alpha}\right)^{n_{\alpha}}$, where $n_{\alpha}$ is a nonzero integer for each $\alpha$ in $\mathscr{A}$.
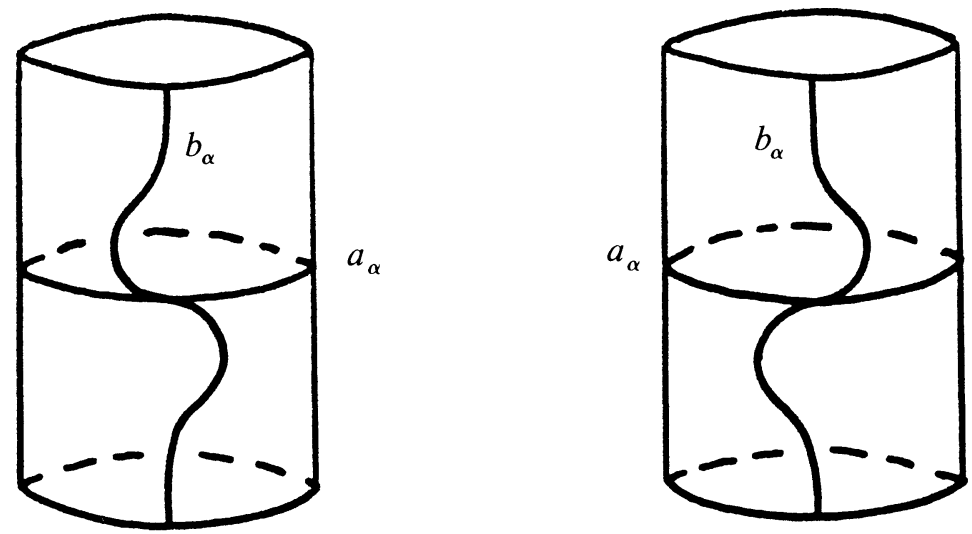

Figure 8

By construction, each train track $T$ associated to our paving looks like one of the two configurations of Figure 5 in an annular neighborhood of any given component of $\mathscr{P}$. Let $\varepsilon(T, \alpha)=-1$ if the local model for $T$ in $A_{\alpha}$ is $T(\alpha,-1)$, and let $\varepsilon(T, \alpha)=1$ if it is $T(\alpha, 1)$. Let $a_{\alpha}$ be the branch of $T$ which runs parallel to $\alpha$, and let $b_{\alpha}$ be either of the two branches which are incident on $\alpha$ (Figure 8).

If $(T, \nu)$ represents $\mathscr{L}$, then we shall simply write $\mathscr{L}=(T, \nu)$. By the properties of a paving, $\nu\left(a_{\alpha}\right)$ and $\nu\left(b_{\alpha}\right)$ do not depend upon the choice of $T$.

Suppose that $\varepsilon(T, \alpha)=-1$ and $\nu\left(a_{\alpha}\right) \geqslant \nu\left(b_{\alpha}\right)$. Then the simple calculation which is carried out in Figure 9 shows:

(1) $\tau_{\alpha}(\mathscr{L}) \in U(T)$,

(2) $\tau_{\alpha}(\nu)\left(a_{\alpha}\right)=\nu\left(a_{\alpha}\right)-\nu\left(b_{\alpha}\right)$,

(3) $\tau_{\alpha}(\nu)(b)=\nu(b)$ for each branch $b \neq a_{\alpha}$.

This calculation is valid because the Dehn twist $\tau_{\alpha}$ is supported on the annular neighborhood. 

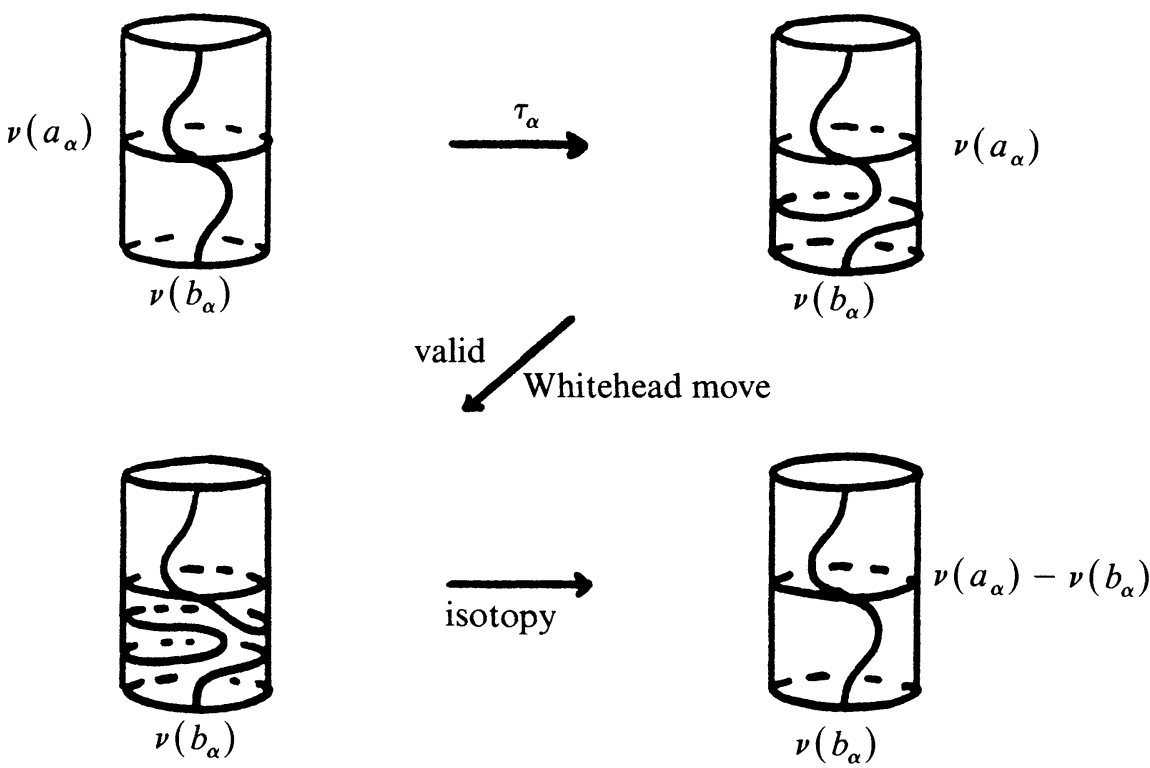

FIGURE 9

Let sign: $\mathbf{R} \rightarrow\{-1,1\}$ be defined by the rule: (a) $\operatorname{sign}(x)=-1$ if $x<0$ and (b) $\operatorname{sign}(x)=1$ if $x \geqslant 0$. We recall that $\tau=\prod_{\alpha \in \mathscr{A}}\left(\tau_{\alpha}\right)^{n_{\alpha}}$. Therefore, since any two of the Dehn twists commute, we have that $\tau^{n}=\prod \tau^{n n_{\alpha}}$ for any nonzero integer $n$.

By calculations similar to the ones carried out in Figure 9, we may deduce the following lemma.

LEMMA 3.4. Let $\mathscr{L} \in U(T), n \in \mathbf{Z}$ and $\varepsilon(\alpha)=\varepsilon(T, \alpha)$. Then:

(1) $\tau^{n}(\mathscr{L}) \in U\left(T^{\prime}\right)$, where $\varepsilon\left(T^{\prime}, \alpha\right)$ is given by the rule

$$
\begin{aligned}
& \varepsilon\left(T^{\prime}, \alpha\right)=\varepsilon(\alpha) \operatorname{sign}\left(\nu\left(a_{\alpha}\right)+n n_{\alpha} \varepsilon(\alpha) \nu\left(b_{\alpha}\right)\right) \quad \text { if } \alpha \in \mathscr{A}, \\
& \varepsilon\left(T^{\prime}, \beta\right)=\varepsilon(\beta) \text { for each component } \beta \text { of } \mathscr{P} \backslash \mathscr{A} .
\end{aligned}
$$

(2) $\tau^{n}(\nu)\left(a_{\alpha}\right)=\left|\nu\left(a_{\alpha}\right)+n n_{\alpha} \varepsilon(\alpha) \nu\left(b_{\alpha}\right)\right|$ if $\alpha \in \mathscr{A}$.

(3) $\tau^{n}(\nu)(b)=\nu(b)$ for each branch $b$ of $T \backslash \mathscr{A}$.

We are now ready to proceed with the proof of the Uniform Convergence Lemma for $\tau$.

Proof. The first step is to construct a function which shall serve as a measure of proximity to Join ${ }_{+}(\tau)$. To this end, if $\mathscr{L}=(T, \nu)$, where $T$ is a track associated to the paving, define $d([\mathscr{L}])=\left(\sum_{\alpha \in \mathscr{A}} \nu\left(b_{\alpha}\right)\right) /|\mathscr{L}|$. By the properties of a paving (Theorem $3.2), d([\mathscr{L}])$ does not depend upon the choice of $T$. Furthermore, it depends only upon the projective class of $\mathscr{L}$. Therefore, it is evident that we have constructed a continuous function

$$
d: \mathscr{P} \mathscr{L}(F) \rightarrow \overline{\mathbf{R}_{+}} .
$$

Since $\mathscr{P} \mathscr{L}(F)$ is compact, there exists an upper bound $C$ for $d$. Furthermore, it is easy to see that

$$
d^{-1}(0)=\operatorname{Join}_{+}(\tau)=\operatorname{Join}_{-}(\tau)
$$


Since $K$ is a compact subset of $\mathscr{P} \mathscr{L}(F)-J_{0 i n}(\tau)$, there exists a positive real $\delta$, such that $d(K) \subset[\delta, C]$. Similarly, there exists a positive real $\varepsilon$, such that

$$
d(\mathscr{P} \mathscr{L}(F) \backslash U) \subset[\varepsilon, C] .
$$

We may assume that $\delta<1$ and $\varepsilon<1$.

Suppose $[\mathscr{L}] \in K$. Then $d([\mathscr{L}]) \geqslant \delta$ and, therefore,

$$
\sum_{\alpha \in \mathscr{A}} \nu\left(b_{\alpha}\right) \geqslant \delta|\mathscr{L}| \text {. }
$$

In order to simplify the notation, we shall let $a_{\alpha}$ denote $\nu\left(a_{\alpha}\right)$, etc.:

$$
|\mathscr{L}|=\sum_{\alpha \in \mathscr{A}} a_{\alpha}+\sum_{b \neq a_{\alpha}} b .
$$

In order to simplify the notation even further, we shall delete the indices of summation. They will be understood in context. By Lemma 3.4,

$$
\begin{aligned}
& \left|\tau^{n} \mathscr{L}\right|=\sum\left|a_{\alpha}+n n_{\alpha} \varepsilon(\alpha) b_{\alpha}\right|+\sum b, \\
& \left|\tau^{n} \mathscr{L}\right| \geqslant \sum n\left|n_{\alpha}\right| b_{\alpha}-\sum a_{\alpha} \geqslant n \sum b_{\alpha}-\sum a_{\alpha}, \\
& \left|\tau^{n} \mathscr{L}\right| \geqslant n \delta|\mathscr{L}|-|\mathscr{L}|=(n \delta-1)|\mathscr{L}| .
\end{aligned}
$$

By definition, $d\left(\left[\tau^{n} \mathscr{L}\right]\right)=\left(\sum \tau^{n}(\nu)\left(b_{\alpha}\right)\right) /\left|\tau^{n} \mathscr{L}\right|$. Thus, by Lemma 3.4, $d\left(\left[\tau^{n} \mathscr{L}\right]\right)=$ $\left(\sum b_{\alpha}\right) /\left|\tau^{n} \mathscr{L}\right|$. Together with (5) we obtain

$$
\begin{array}{ll}
\text { If } n>1 / \delta, & \text { then } d\left(\left[\tau^{n} \mathscr{L}\right]\right) \leqslant\left(\sum b_{\alpha}\right) /((n \delta-1)|\mathscr{L}|), \\
& \text { or } d\left(\left[\tau^{n} \mathscr{L}\right]\right) \leqslant d([\mathscr{L}]) /(n \delta-1) .
\end{array}
$$

Therefore, we conclude that if $n>1 / \delta$, then

$$
d\left(\left[\tau^{n} \mathscr{L}\right]\right) \leqslant C /(n \delta-1) .
$$

The result follows easily from (7) and the previous remarks.

4. The Uniform Convergence Lemma for pseudo-Anosovs. This section follows the same development as the previous section. Only, at this point, we consider a fixed pseudo-Anosov map $\tau$ on a fixed surface $F$. There exists a positive integer $M$, such that $\tau^{M}$ preserves each component of $F$.

We recall that $\mathscr{M} \mathscr{L}(F)$ is naturally homeomorphic to $\prod_{i \in I} \mathscr{M} \mathscr{L}\left(F_{i}\right)$. Hence, if $\mathscr{L}_{i} \in \mathscr{M} \mathscr{L}\left(F_{i}\right)$, we may write $\left(\mathscr{L}_{i}\right)_{i \in I} \in \mathscr{M} \mathscr{L}(F)$. If $A_{i} \subset \mathscr{P} \mathscr{L}\left(F_{i}\right)$, then we define the join of $\left\{A_{i} \mid i \in I\right\},{ }_{i \in I} A_{i}$, by the rule

$$
\underset{i \in I}{*} A_{i}=\left\{[\mathscr{L}] \in \mathscr{P} \mathscr{L}(F) \mid \mathscr{L}=\left(\mathscr{L}_{j}\right)_{j \in I},\left[\mathscr{L}_{i}\right] \in A_{i}, i \in I\right\}
$$

In this case, we may define Join ${ }_{ \pm}(\tau)=*_{i \in I}\left\{\left[\mathscr{L}_{i \pm}\right]\right\}$, where $\left[\mathscr{L}_{i+}\right]$ is the attracting lamination for $\tau_{i}$, and $\left[\mathscr{L}_{i-}\right]$ is the repelling lamination. As in the previous section, it suffices to establish the U.C.L. for $\tau^{M}$. Henceforth, we assume that $M=1$.

The projective laminations $\left\{\left[\mathscr{L}_{ \pm}\right]\right\}$for a pseudo-Anosov mapping class on a connected surface have some striking properties which we will find useful.

LEMMA 4.1 [Th3]. Let $F_{i}$ be a connected surface, $\tau_{i}$ a pseudo-Anosov mapping class on $F_{i}$, and $\left\{\left[\mathscr{L}_{+}\right],\left[\mathscr{L}_{-}\right]\right\}$the corresponding pair of projective laminations $\left\{\left[\left(\gamma_{+}, \mu_{+}\right)\right]\right.$, $\left.\left[\left(\gamma_{-}, \mu_{-}\right)\right]\right\}$for $\tau_{i}$. 
(1) $\gamma_{ \pm}$is uniquely ergodic. That is, if $\mathscr{L}$ is a measured lamination and $\operatorname{supp}(\mathscr{L})=\gamma_{ \pm}$, then $[\mathscr{L}]=\left[\mathscr{L}_{ \pm}\right]$.

(2) $\gamma_{ \pm}$is minimal. That is, if $\gamma$ is a geodesic lamination and $\gamma \subset \gamma_{ \pm}$, then either $\gamma=\varnothing$ or $\gamma=\gamma_{ \pm}$.

(3) $\gamma_{ \pm}$is maximal among supports of measured laminations. That is, if $\mathscr{L}$ is a measured lamination $(\gamma, \mu)$ and $\gamma_{ \pm} \subset \gamma$, then $\gamma_{ \pm}=\gamma$.

In addition to the tools already discussed we shall need to employ Thurston's intersection form $\langle\rangle:, \mathscr{M} \mathscr{L}(F) \times \mathscr{M} \mathscr{L}(F) \rightarrow \overline{\mathbf{R}_{+}}$. The reader is referred to [Ke, Th1, Th5] for expositions of this form. We shall give a brief description.

Suppose $\mathscr{L}_{1}$ and $\mathscr{L}_{2}$ are measured laminations $\left(\gamma_{1}, \mu_{1}\right)$ and $\left(\gamma_{2}, \mu_{2}\right)$ on $F$. At a point $p$ of transverse intersection of $\gamma_{1}$ and $\gamma_{2}$, we may find a chart $\phi: U(p) \rightarrow(a, b)$ $\times(c, d)$, where $U(p)$ is a neighborhood of $p$ and $(a, b)$ and $(c, d)$ are intervals. We can choose this chart so that $\phi\left(\mathscr{L}_{1} \cap U(p)\right)=(a, b) \times Y$ and $\phi\left(\mathscr{L}_{2} \cap U(p)\right)=X$ $\times(c, d)$, where $Y$ and $X$ are closed subsets of $(c, d)$ and $(a, b)$ respectively (Figure $10)$.

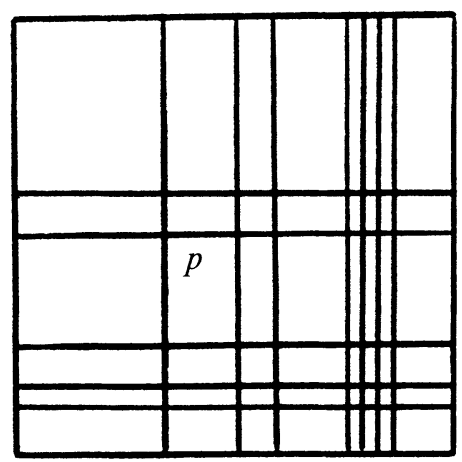

Figure 10

$\mu_{2}$ induces a Borel measure on $(a, b)$ and $\mu_{1}$ induces a Borel measure on $(c, d)$. The product $\mu_{1} \otimes \mu_{2}$ is a finite Borel measure on $U(p)$ supported on the transverse intersection $\left(\gamma_{1} \pitchfork \gamma_{2}\right) \cap U(p)$. These local measures are compatible and one obtains a Borel measure $\mu_{1} \otimes \mu_{2}$ on $F$, supported on $\gamma_{1} \pitchfork \gamma_{2}$, and of finite total mass $\int_{F} d\left(\mu_{1} \otimes \mu_{2}\right)$. The intersection form is defined by the rule

$$
\left\langle\mathscr{L}_{1}, \mathscr{L}_{2}\right\rangle=\int_{F} d\left(\mu_{1} \otimes \mu_{2}\right) \text {. }
$$

TheOREM 4.2 (ThuRston [Th5]). $\langle\rangle:, \mathscr{M} \mathscr{L}(F) \times \mathscr{M} \mathscr{L}(F) \rightarrow \overline{\mathbf{R}_{+}}$is continuous.

REMARK. Thurston stated this theorem for $\mathscr{M}_{0}(F)(\S 6)$. With a few technical alterations, the proof actually establishes the stronger result. Furthermore, the result was formulated for connected surfaces. By an obvious argument, one may obtain the result for surfaces of several components (see $\S 1$ ).

If $\mathscr{L}$ and $\mathscr{L}^{\prime}$ are measured laminations $(\gamma, \mu)$ and $\left(\gamma^{\prime}, \mu^{\prime}\right)$ such that $\gamma \pitchfork \gamma^{\prime}=\varnothing$, then we can define the sum of $\mathscr{L}$ and $\mathscr{L}^{\prime}, \mathscr{L}+\mathscr{L}^{\prime}$, by the rule

$$
\mathscr{L}+\mathscr{L}^{\prime}=\left(\gamma \cup \gamma^{\prime}, \mu+\mu^{\prime}\right) \text {. }
$$


The local transverse measures of $\mu+\mu^{\prime}$ are just the sums of the local transverse measures of $\mu$ and $\mu^{\prime}$. Using this partial addition on $\mathscr{M} \mathscr{L}(F)$, we may define a partial ordering on $\mathscr{M} \mathscr{L}(F)$ by the rule

$$
\mathscr{L}^{\prime} \geqslant \mathscr{L} \text { if and only if } \mathscr{L}^{\prime}=\mathscr{L}+\mathscr{L}^{\prime \prime} \text { for some } \mathscr{L}^{\prime \prime} \in \mathscr{M} \mathscr{L}(F) .
$$

In addition to the previous theorem, we have the following simple observations.

LeMma 4.3. Let $\mathscr{L}_{1}, \mathscr{L}_{2}, \mathscr{L} \in \mathscr{M} \mathscr{L}(F), \tau \in \Gamma(F)$ and $\lambda \in \overline{\mathbf{R}_{+}}$. Then:

(1) $\left\langle\mathscr{L}_{1}, \mathscr{L}_{2}\right\rangle=\left\langle\mathscr{L}_{2}, \mathscr{L}_{1}\right\rangle$,

(2) $\left\langle\tau \mathscr{L}_{1}, \tau \mathscr{L}_{2}\right\rangle=\left\langle\mathscr{L}_{1}, \mathscr{L}_{2}\right\rangle$,

(3) $\left\langle\lambda \mathscr{L}_{1}, \mathscr{L}_{2}\right\rangle=\lambda\left\langle\mathscr{L}_{1}, \mathscr{L}_{2}\right\rangle$,

(4) if $\mathscr{L}_{1}+\mathscr{L}_{2}$ is defined, then $\left\langle\mathscr{L}_{1}+\mathscr{L}_{2}, \mathscr{L}\right\rangle=\left\langle\mathscr{L}_{1}, \mathscr{L}\right\rangle+\left\langle\mathscr{L}_{2}, \mathscr{L}\right\rangle$, and

(5) if $\mathscr{L}_{1} \geqslant \mathscr{L}_{2}$, then $\left\langle\mathscr{L}_{1}, \mathscr{L}\right\rangle \geqslant\left\langle\mathscr{L}_{2}, \mathscr{L}\right\rangle$.

The following lemma allows us to construct functions which will serve as measures of proximity to $\operatorname{Join}_{ \pm}(\tau)$.

LEMMA 4.4. There exists a pair of measured laminations $\left\{\mathscr{L}_{+}, \mathscr{L}_{-}\right\}$and a positive real number $\lambda>1$, such that if $[\mathscr{L}] \in \mathscr{P} \mathscr{L}(F)$, then:

(1) $\tau\left(\mathscr{L}_{+}\right) \geqslant \lambda \cdot \mathscr{L}_{+}$and $\tau\left(\mathscr{L}_{-}\right) \leqslant \lambda^{-1} \cdot \mathscr{L}_{-}$,

(2) $\left\langle\mathscr{L}, \mathscr{L}_{+}\right\rangle=0$ if and only if $[\mathscr{L}] \in \mathrm{Join}_{+}(\tau)$,

(3) $\left\langle\mathscr{L}, \mathscr{L}_{-}\right\rangle=0$ if and only if $[\mathscr{L}] \in$ Join_$_{-}(\tau)$.

ProOF. For each connected component $F_{i}$ of $F$, consider the pair of projective laminations $\left\{\left[\mathscr{L}_{i+}\right],\left[\mathscr{L}_{i-}\right]\right\}$ and the real number $\lambda_{i}>1$, such that

$$
\tau_{i}\left(\mathscr{L}_{i+}\right)=\lambda_{i} \cdot \mathscr{L}_{i+} \quad \text { and } \tau_{i}\left(\mathscr{L}_{i-}\right)=\lambda_{i}^{-1} \cdot \mathscr{L}_{i-} .
$$

(1) Let $\mathscr{L}_{+}=\sum_{i \in I} \mathscr{L}_{i+}, \mathscr{L}_{-}=\sum_{i \in I} \mathscr{L}_{i-}$ and $\lambda=\min \left\{\lambda_{i} \mid i \in I\right\}$.

(2) We can assume $F$ is connected. With this assumption in mind, suppose that $\left\langle\mathscr{L}, \mathscr{L}_{+}\right\rangle=0$. Then $\gamma \pitchfork \gamma_{+}=\varnothing$, and $\mathscr{L}+\mathscr{L}_{+}$is defined. By Lemma 4.1(3), $\gamma_{+}=\gamma$ $\cup \gamma_{+}$and $\gamma \subset \gamma_{+}$. Thus by Lemma 4.1(2), $\gamma=\gamma_{+}$. Finally, by Lemma 4.1(1), $[\mathscr{L}]=\left[\mathscr{L}_{+}\right]$. The converse is trivial.

(3) This assertion follows in the same manner.

REMARK. If $F$ is not connected, then the projective classes $\left[\mathscr{L}_{+}\right]$and $\left[\mathscr{L}_{-}\right]$are not uniquely determined by Lemma 4.4 .

We now give the proof of the Uniform Convergence Lemma for $\tau$.

Proof. Define a pair of continuous functions

$$
j_{ \pm}: \mathscr{P} \mathscr{L}(F) \backslash \text { Join }_{\mp}(\tau) \rightarrow \overline{\mathbf{R}_{+}}
$$

by the rules

$$
j_{ \pm}([\mathscr{L}])=\left\langle\mathscr{L}, \mathscr{L}_{ \pm}\right\rangle /\left\langle\mathscr{L}, \mathscr{L}_{\mp}\right\rangle
$$

By Lemma 4.4, these rules are defined on the domains indicated above. The fact that the rules determine well-defined continuous functions follows from the homogeneity of $\langle$,$\rangle and Theorem 4.2. Furthermore, by Lemma 4.4, it is easy to see that$

$$
j_{ \pm}^{-1}(0)=\operatorname{Join}_{ \pm}(\tau) \text {. }
$$


Finally, from the definition, it is clear that

$$
\begin{aligned}
& \text { If }[\mathscr{L}] \in \mathscr{P} \mathscr{L}(F) \backslash\left(\operatorname{Join}_{-}(\tau) \cup \operatorname{Join}_{+}(\tau)\right), \\
& \text { then } j_{+}([\mathscr{L}]) \cdot j_{-}([\mathscr{L}])=1 .
\end{aligned}
$$

Since $K$ is compact, there exists a positive real $C$, such that $j_{+}(K) \subset[0, C]$. Similarly, there exists a positive real $D$, such that

$$
j_{-}(\mathscr{P} \mathscr{L}(F) \backslash U) \subset[0, D] .
$$

Suppose that $[\mathscr{L}] \in K$. Since $[\mathscr{L}] \notin$ Join_ $(\tau)$, it follows from the definition that $\tau^{n}([\mathscr{L}]) \notin$ Join $_{-}(\tau)$. Thus, we may write the following string of inequalities:

$$
\begin{aligned}
j_{+}\left(\tau^{n}([\mathscr{L}])\right) & =\left\langle\tau^{n} \mathscr{L}, \mathscr{L}_{+}\right\rangle /\left\langle\tau^{n} \mathscr{L}, \mathscr{L}_{-}\right\rangle=\left\langle\mathscr{L}, \tau^{-n} \mathscr{L}_{+}\right\rangle /\left\langle\mathscr{L}, \tau^{-n} \mathscr{L}_{-}\right\rangle \\
& \leqslant\left\langle\mathscr{L}, \lambda^{-n} \mathscr{L}_{+}\right\rangle /\left\langle\mathscr{L}, \lambda^{n} \mathscr{L}_{-}\right\rangle \leqslant \lambda^{-2 n}\left\langle\mathscr{L}, \mathscr{L}_{+}\right\rangle /\left\langle\mathscr{L}, \mathscr{L}_{-}\right\rangle \\
& \leqslant \lambda^{-2 n} j_{+}([\mathscr{L}]) .
\end{aligned}
$$

Choose a positive integer $N$, such that if $n \geqslant N$, then $\lambda^{-2 n}<1 /(2 C D)$. Then if $n \geqslant N$

$$
j_{+}\left(\tau^{n}([\mathscr{L}])\right)<C /(2 C D)=1 /(2 D) .
$$

We may assume that $\tau^{n}([\mathscr{L}]) \notin \operatorname{Join}_{+}(\tau)$. Therefore, $j_{-}\left(\tau^{n}([\mathscr{L}])\right)>2 D$. By the choice of $D$, we conclude that $\tau^{n}([\mathscr{L}]) \in U$.

5. The Uniform Convergence Lemma for simple direct sums. Suppose $F=F_{1} \amalg F_{2}$, $F_{1} \neq \varnothing \neq F_{2}$, and $\tau$ is a mapping class in $\Gamma(F)$ which preserves $F_{1}$ and $F_{2}$. If $\tau_{1}$, the restriction of $\tau$ to $F_{1}$, is of algebraically finite type and $\tau_{2}$, the restriction of $\tau$ to $F_{2}$, is pseudo-Anosov, then we say that $\tau$ is a simple direct sum. In this section, we shall prove the Uniform Convergence Lemma for simple direct sums. Henceforth, throughout this section, $\tau$ denotes a fixed simple direct sum on a fixed surface $F$. There exists a positive integer $M$, such that $\tau_{1}^{M}$ is a product of Dehn twists about the components of $\mathscr{A}_{\tau}$, and $\tau_{2}^{M}$ preserves the components of $F_{2}$.

In this case, we may define Join ${ }_{+}(\tau)$ to be $\operatorname{Join}_{+}\left(\tau_{1}\right) * \operatorname{Join}_{+}\left(\tau_{2}\right)$. As in the previous two sections, therefore, it suffices to establish the U.C.L. for $\tau^{M}$. Henceforth, we assume that $M=1$.

The proof will follow the strategy which was used in $\S \S 3$ and 4 . We will construct functions which measure proximity to the Joins and then use these to prove the uniformity of convergence. At this point, we introduce a paving adapted to $\mathscr{A}$, the essential reduction system for $\tau$. For each integer $i=1$ or 2 , this paving restricts to a paving of $\mathscr{P} \mathscr{L}\left(F_{i}\right)$ adapted to $\mathscr{A}_{i}$, the essential reduction system for $\tau_{i}$.

We recall the bounded continuous function which was defined in $\S 3$, where $C$ denotes a bound

$$
d: \mathscr{P} \mathscr{L}\left(F_{1}\right) \rightarrow[0, C] \quad \text { such that } d^{-1}(0)=\operatorname{Join}_{ \pm}\left(\tau_{1}\right) .
$$

There exists a pair of bounded continuous functions

$$
e_{ \pm}: \mathscr{P} \mathscr{L}\left(F_{2}\right) \rightarrow[0, C] \text { such that } e_{ \pm}^{-1}(0)=\operatorname{Join}_{ \pm}\left(\tau_{2}\right) .
$$

The functions are defined by the rules

$$
e_{+}([\mathscr{L}])=\left\langle\mathscr{L}, \mathscr{L}_{+}\right\rangle /|\mathscr{L}| \text { and } e_{-}([\mathscr{L}])=\left\langle\mathscr{L}, \mathscr{L}_{-}\right\rangle /|\mathscr{L}|,
$$

where $\mathscr{L}_{+}$and $\mathscr{L}_{-}$are given by Lemma 4.4 applied to $\tau_{2}$. By the usual arguments, 
these rules determine well-defined, bounded continuous functions. The conditions on $e_{+}^{-1}(0)$ follow from Lemma 4.4 .

We shall construct a corresponding function for $\tau$ and its Joins. We will then establish the Uniform Convergence Lemma for $\tau$ in much the same manner as we did in $\$ 3$ for mapping classes of algebraically finite type. In order to accomplish this, however, we shall require more explicit information regarding the rates of convergence of the functions $d$ and $e_{ \pm}$. The following series of lemmas provide this information.

LEMMA 5.1. There exists a positive real $B_{1}$, such that $\left|\tau_{1}^{n} \mathscr{L}\right| \leqslant|n| B_{1}|\mathscr{L}|$ for each measured lamination $\mathscr{L} \in \mathscr{M} \mathscr{L}\left(F_{1}\right)$ and each nonzero integer $n$.

ProOF. (We shall adopt the same simplifications of notation as in §3.)

(1) $\left|\tau_{1}^{n} \mathscr{L}\right|=\sum b+\sum\left|a_{\alpha}+n n_{\alpha} \varepsilon(\alpha) b_{\alpha}\right|$.

(2) $\left|\tau_{1}^{n} \mathscr{L}\right| \leqslant \sum b+\sum a_{\alpha}+|n| \sum\left|n_{\alpha}\right| b_{\alpha}$.

Let $n_{0}=\operatorname{maximum}\left\{\left|n_{\alpha}\right| \mid \alpha \in \mathscr{A}\right\}$.

(3) $\left|\tau_{1}^{n} \mathscr{L}\right| \leqslant \Sigma b+\sum a_{\alpha}+|n| n_{0} \sum b_{\alpha}$.

Since $\sum b_{\alpha} \leqslant C|\mathscr{L}|$ we conclude:

(4) $\left|\tau_{1}^{n} \mathscr{L}\right| \leqslant|\mathscr{L}|+|n| n_{0} C|\mathscr{L}| \leqslant|n|\left(1+n_{0} C\right)|\mathscr{L}|$.

Lemma 5.2. Let $\delta, \varepsilon>0$. Then there exists a positive integer $M_{1}$, such that if $d([\mathscr{L}]) \geqslant \delta$ and $n \geqslant M_{1}$, then

(a) $d\left(\left[\tau_{1}^{n} \mathscr{L}\right]\right)<\varepsilon$ and

(b) $\left|\tau_{1}^{n+1} \mathscr{L}\right| \geqslant(1-\varepsilon)\left|\tau_{1}^{n} \mathscr{L}\right|$.

Proof. Define the following function:

$$
\begin{aligned}
\rho_{1}: \mathscr{P} \mathscr{L}\left(F_{1}\right) & \rightarrow \mathbf{R}_{+} \\
{[\mathscr{L}] } & \rightarrow\left|\tau_{1} \mathscr{L}\right| /|\mathscr{L}| .
\end{aligned}
$$

Clearly, $\rho_{1}$ is continuous. Furthermore, if $[\mathscr{L}] \in \operatorname{Join}_{+}\left(\tau_{1}\right)$, then $\tau_{1} \mathscr{L} \geqslant \mathscr{L}$ and $\rho_{1}([\mathscr{L}]) \geqslant 1$. Therefore, $\rho_{1}^{-1}((1-\varepsilon, \infty))$ is an open neighborhood of $\operatorname{Join}_{+}\left(\tau_{1}\right)$. The same statement holds for $d^{-1}((0, \varepsilon))$, and by similar reasoning $d^{-1}([\delta, C])$ is a compact subset of $\mathscr{P} \mathscr{L}(F) \backslash$ Join_ $\left(\tau_{1}\right)$.

The lemma follows by applying the U.C.L. for $\tau_{1}$.

Lemma 5.3. There exists a positive real $B_{2}$, such that $\left|\tau_{2}^{n}(\mathscr{L})\right| \leqslant B_{2}^{|n|}|\mathscr{L}|$ for each measured lamination $\mathscr{L} \in \mathscr{M} \mathscr{L}\left(F_{2}\right)$ and each nonzero integer $n$.

Proof. Let $\rho_{2}: \mathscr{P} \mathscr{L}\left(F_{2}\right) \rightarrow \mathbf{R}_{+}$be defined as $\rho_{1}$ in the proof of Lemma 5.2. Since $\mathscr{P} \mathscr{L}\left(F_{2}\right)$ is compact, there exists a positive real $E_{+}$, such that $\rho_{2}$ is bounded by $E_{+}$. Hence, $\left|\tau_{2}(\mathscr{L})\right| \leqslant E_{+}|(\mathscr{L})|$. Likewise, there exists a positive real $E_{-}$, such that $\left|\tau_{2}^{-1}(\mathscr{L})\right| \leqslant E_{-}|\mathscr{L}|$. The lemma is satisfied by $B_{2}$, where $B_{2}=\max \left\{E_{-}, E_{+}\right\}$.

For the next two lemmas, let $D$ denote a positive number such that $1<D<\lambda$, where $\lambda$ is given by Lemma 4.4 applied to $\tau_{2}$.

LEMMA 5.4. Let $\delta, \varepsilon>0$. Then there exists a positive integer $M_{2}$, such that if $e_{-}([\mathscr{L}]) \geqslant \delta$ and $n \geqslant M_{2}$, then

(c) $d\left(\left[\tau_{2}^{n} \mathscr{L}\right]\right)<\varepsilon$ and

(d) $\left|\tau_{2}^{n+1}(\mathscr{L})\right| \geqslant D\left|\tau_{2}^{n}(\mathscr{L})\right|$. 
PROOF. The proof is similar to the proof of Lemma 5.2.

LEMMA 5.5. For each positive real number $\varepsilon$, there exists a positive integer $Q$, such that if $n \geqslant Q$, then either

(1) $e_{+}\left(\left[\tau_{2}^{n} \mathscr{L}\right]\right)<\varepsilon$, or

(2) $\left|\tau_{2}^{n-Q}(\mathscr{L})\right| \leqslant D^{-(n-Q)}|\mathscr{L}|$.

Proof. Let $U=\rho_{2}^{-1}\left(\left(0, D^{-1}\right)\right)$ and $K=\rho_{2}^{-1}\left(\left[0, D^{-1}\right]\right)$. By the U.C.L. for pseudoAnosovs applied to $\tau_{2}^{-1}$, there is a positive integer $N$, such that if $n \geqslant N$, then $\tau_{2}^{-n}(K) \subset U$. Let $U_{0}=\bigcup_{n \geqslant N} \tau_{2}^{-n}(U)$. We easily conclude that Join_ $\left(\tau_{2}\right) \subset U_{0} \subset U$, $\tau_{2}^{-1}\left(U_{0}\right) \subset U_{0}$, and if $\mathscr{L} \in U_{0}$, then $\left|\tau_{2} \mathscr{L}\right|<D^{-1}|\mathscr{L}|$.

By a second application of the U.C.L. for pseudo-Anosovs, there exists a positive integer $Q$, such that $\tau_{2}^{Q}\left(\mathscr{P} \mathscr{L}\left(F_{2}\right) \backslash U_{0}\right) \subset e_{+}^{-1}(0, \varepsilon)$. Hence, if $n \geqslant Q$ and $e_{+}\left(\left[\tau_{2}^{n} \mathscr{L}\right]\right) \geqslant$ $\varepsilon$, then $\left[\tau_{2}^{n-Q}(\mathscr{L})\right] \in U_{0}$. It follows easily that $\left|\tau_{2}^{n-Q}(\mathscr{L})\right| \leqslant D^{-(n-Q)}|\mathscr{L}|$.

We are now ready to begin the proof of the Uniform Convergence Lemma for $\tau$.

Proof. From the functions given above, we may construct corresponding functions for $\tau$.

$$
f_{ \pm}: \mathscr{P} \mathscr{L}(F) \rightarrow[0, C] \quad \text { such that } f_{ \pm}^{-1}(0)=\operatorname{Join}_{ \pm}(\tau) .
$$

By convention, define $d(0)=e_{+}(0)=0$. Using these conventions, we define

$$
f_{ \pm}\left(\left[\mathscr{L}_{1} \oplus \mathscr{L}_{2}\right]\right)=\left(\left|\mathscr{L}_{1}\right| /\left|\mathscr{L}_{\mid}\right|\right) d\left(\left[\mathscr{L}_{1}\right]\right)+\left(\left|\mathscr{L}_{2}\right| /|\mathscr{L}|\right) e_{ \pm}\left(\left[\mathscr{L}_{2}\right]\right)
$$

By the standard arguments employed previously, this determines a pair of well-defined, bounded continuous functions. The condition on $f_{ \pm}^{-1}(0)$ follows from the definition of the Joins.

As in the proof of the U.C.L. for mapping classes of algebraically finite type, it suffices to prove that for each pair of positive reals $\delta$ and $\varepsilon$, there exists a positive integer $N$, such that if $n \geqslant N$ and $f_{-}([\mathscr{L}]) \geqslant \delta$, then $f_{+}\left(\tau^{n}([\mathscr{L}])\right)<\varepsilon$.

Suppose, therefore, that $f_{-}([\mathscr{L}]) \geqslant \delta$. Then, either $d\left(\left[\mathscr{L}_{1}\right]\right) \geqslant \delta / 2$ or $e_{-}\left(\left[\mathscr{L}_{2}\right]\right) \geqslant$ $\delta / 2$. Suppose that $d\left(\left[\mathscr{L}_{1}\right]\right) \geqslant \delta / 2$. By Lemma 5.2 , there exists a positive integer $M_{1}$, such that if $m \geqslant M_{1}$, then

(a) $d\left(\left[\tau_{1}^{m} \mathscr{L}_{1}\right]\right)<\varepsilon / 2$ and

(b) $\left(\left[\tau_{1}^{m+1} \mathscr{L}_{1}\right]\right) \geqslant(1-\varepsilon)\left(\left[\tau_{1}^{m} \mathscr{L}_{1}\right]\right)$.

Similarly, if $e_{-}\left(\left[\mathscr{L}_{2}\right]\right) \geqslant \delta / 2$, then, by Lemma 5.4 there exists a positive integer $M_{2}$, such that if $m \geqslant M_{2}$, then

(c) $e_{+}\left(\left[\tau_{2}^{m} \mathscr{L}_{2}\right]\right)<\varepsilon / 2$ and

(d) $\left(\left[\tau_{2}^{m+1} \mathscr{L}_{2}\right]\right) \geqslant D\left(\left[\tau_{2}^{m} \mathscr{L}_{2}\right]\right)$.

At this point let us assume that $\delta>1, \varepsilon<1$ and $1<D(1-\varepsilon)$. (This last assumption is possible, since $D>1$.) Let $M=\max \left(M_{1}, M_{2}\right)$.

Case $1 . \mathscr{L}_{1}=0$. In this case, $e_{-}\left(\left[\mathscr{L}_{2}\right]\right) \geqslant \delta / 2$. It is easy to see from (c) and the definition of $f_{+}$, that if $m \geqslant M, f_{+}\left(\left[\tau^{m} \mathscr{L}\right]\right)<\varepsilon$.

Case 2. $\mathscr{L}_{2}=0$. By the same token, if $m \geqslant M, f_{+}\left(\left[\tau^{m} \mathscr{L}\right]\right)<\varepsilon$. Henceforth, we assume that $\mathscr{L}_{1} \neq 0 \neq \mathscr{L}_{2}$.

Case 3. $d\left(\left[\mathscr{L}_{1}\right]\right) \geqslant \delta / 2$ and $e_{-}\left(\left[\mathscr{L}_{2}\right]\right) \geqslant \delta / 2$. Again, if $m \geqslant M$, then $f_{+}\left(\left[\tau^{m} \mathscr{L}\right]\right)<\varepsilon$.

We now arrive at the difficult cases. It is here that Lemmas 5.1-5.5 are crucial.

Case 4. $d\left(\left[\mathscr{L}_{1}\right]\right) \geqslant \delta / 2$ and $e_{-}\left(\left[\mathscr{L}_{2}\right]\right)<\delta / 2$. By Lemma 5.5 , choose a positive integer $Q$, such that if $n \geqslant Q$, then either

$$
e_{+}\left(\left[\tau_{2}^{n} \mathscr{L}_{2}\right]\right)<\varepsilon / 2
$$




$$
\left|\tau_{2}^{n-Q} \mathscr{L}_{2}\right| \leqslant D^{Q}\left|\mathscr{L}_{2}\right| / D^{n} .
$$

Let $Q_{1}=\max \left(M_{1}, Q\right)$. By (a) above, if $n \geqslant Q_{1}$ and (6) holds, then $f_{+}\left(\left[\tau^{n} \mathscr{L}\right]\right)<\varepsilon$. Henceforth, we assume that $n \geqslant Q_{1}$ and (7) holds. From the definition of $f_{-}$, and the assumptions that $f_{-}([\mathscr{L}]) \geqslant \delta$ and $e_{-}\left(\left[\mathscr{L}_{2}\right]\right)<\delta / 2$, we observe that

$$
\delta \leqslant\left(\left|\mathscr{L}_{1}\right| /\left|\mathscr{L}_{2}\right|\right) C+\delta / 2 .
$$

In the following argument, the constants will become rather cumbersome. We shall simply write all irrelevant constants by the same symbol, $k$.

If $n \geqslant Q_{1}$, then

(i) $\left|\tau_{2}^{n} \mathscr{L}_{2}\right| \leqslant k\left|\tau_{2}^{n-Q} \mathscr{L}_{2}\right|$ by Lemma 5.3 .

(ii) $\left|\tau_{2}^{n} \mathscr{L}_{2}\right| \leqslant k\left|\mathscr{L}_{2}\right| / D^{n}$ by (6).

(iii) $\left|\tau_{2}^{n} \mathscr{L}_{2}\right| \leqslant k\left|\mathscr{L}_{1}\right| / D^{n}$ by (7).

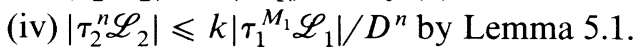

(v) $\left|\tau_{2}^{n} \mathscr{L}_{2}\right| \leqslant k\left|\tau_{1}^{n} \mathscr{L}_{1}\right| /\left(D^{n}(1-\varepsilon)^{n-M_{1}}\right)$ by (b).

(vi) $\left|\tau_{2}^{n} \mathscr{L}_{2}\right| \leqslant k\left|\tau_{1}^{n} \mathscr{L}_{1}\right| /(D(1-\varepsilon))^{n}$.

Clearly, by (vi), there exists $N_{1}$, such that $N_{1} \geqslant Q_{1}$ and if $n \geqslant N_{1}$, then (vii) $\left|\tau_{2}^{n} \mathscr{L}_{2}\right| \leqslant \varepsilon\left|\tau_{1}^{n} \mathscr{L}_{1}\right| / 2 C$.

It is easy to see that if $n \geqslant N_{1}$, then $f_{+}\left(\left[\tau^{n} \mathscr{L}\right]\right)<\varepsilon$.

Case 5. $d\left(\left[\mathscr{L}_{1}\right]\right)<\delta / 2$ and $e_{-}\left(\left[\mathscr{L}_{2}\right]\right) \geqslant \delta / 2$. This case follows in a similar manner. Since we have covered all cases, the proof is complete.

6. Projective lamination spaces and reduction maps. Suppose that $\tau$ is an arbitrary mapping class on $F$. Let $\mathscr{A}$ denote the essential reduction system of $\tau$. Consider the subcollection $\mathscr{B}$ of simple closed curves which, after reduction along $\mathscr{A}$, correspond to boundary components of components of $\hat{F}$ for which the restriction of $\hat{\tau}$ is pseudo-Anosov. Clearly, $\mathscr{B}$ is a reduction system for $\tau$.

It is easy to see that the reduction of $\tau$ along $\mathscr{B}$ is either of algebraically finite type (in which case, $\mathscr{B}=\varnothing$ and $\tau$ is of algebraically finite type), or pseudo-Anosov (in which case, $\mathscr{B}=\mathscr{A}$ ), or a simple direct sum. In any event, the reduction of $\tau$ along $\mathscr{B}, \hat{\tau}$, satisfies the Uniform Convergence Lemma by the results of the previous three sections.

From this simple observation, it is natural to compare the action of $\tau$ on $\mathscr{P} \mathscr{L}(F)$ with the action of $\hat{\tau}$ on $\mathscr{P} \mathscr{L}(\hat{F})$. Therefore, in this section, we shall construct reduction maps, natural maps between the projective lamination spaces of surfaces and their associated reductions. In the next section, we shall employ these maps to give the general definition of the Joins of a mapping class. And then, in $\S 8$, we shall employ them to derive the Uniform Convergence Lemma for an arbitrary mapping class in the manner suggested above.

Suppose that $F$ is a surface, $\mathscr{A}$ is an admissible system on $F$ and $\hat{F}=F \backslash \mathscr{A}$ as in $\S 2$. Our objective is to construct a continuous reduction map

$$
\Lambda: \mathscr{M} \mathscr{L}(F) \rightarrow \mathscr{M} \mathscr{L}(\hat{F}) .
$$



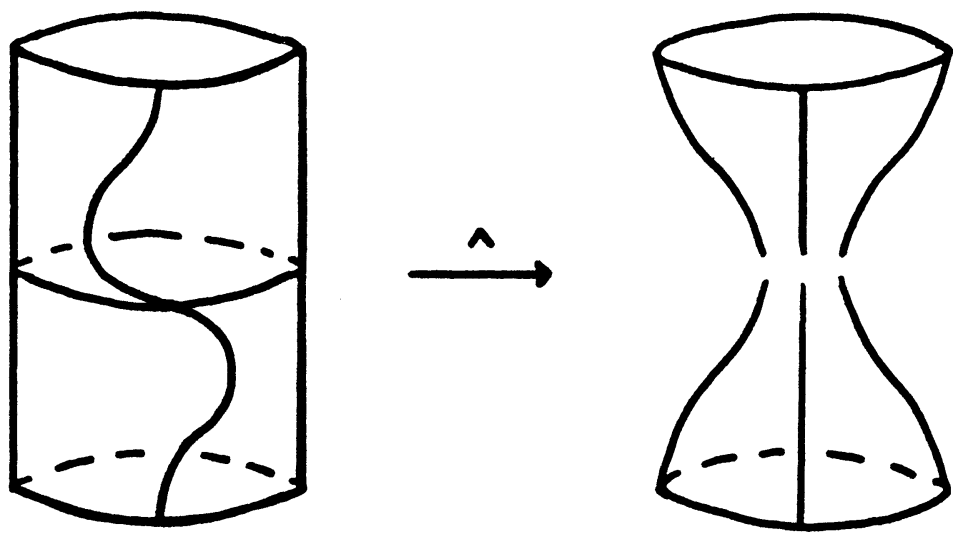

Figure 11

Intuitively, one may think of this map as given by the rule

$$
(\gamma, \mu) \mapsto\left(\gamma \cap \hat{F},\left.\mu\right|_{\gamma \cap \hat{F}}\right) .
$$

Unfortunately, $\gamma \cap \hat{F}$ is not a geodesic lamination (see Note, §2). It appears that $\gamma \cap \hat{F}$ is isotopic to a geodesic lamination and one could use such an isotopy to supply this geodesic lamination with a transverse measure induced from $\mu$. We shall not attempt to carry this program out. Instead we shall define our maps in terms of measured train tracks.

Choose a pants decomposition $\mathscr{P}$ of $F$ such that $\mathscr{A} \subset \mathscr{P}$. Let $\mathscr{P}$ be the corresponding paving of $\mathscr{M} \mathscr{L}(F)$. We are going to build directly a paving of $\mathscr{M} \mathscr{L}(\hat{F})$ from this paving of $\mathscr{M} \mathscr{L}(F)$. The notation for the associated objects for $\mathscr{M} \mathscr{L}(F)$ will be as in $\S 3$. The corresponding objects for $\mathscr{M} \mathscr{L}(\hat{F})$ will be denoted likewise except for a superscript, ^.

Let $\hat{\mathscr{A}}$ be the collection of boundary components of annuli corresponding to the components of $\mathscr{A}$. Let $\hat{\mathscr{P}}=\mathscr{P} \backslash \mathscr{A}$ be considered as a family of simple closed curves on $\hat{F}$.

We may choose a complete hyperbolic structure on $\hat{F}$ in which $\hat{\mathscr{P}}$ is a family of simple closed geodesics. In this structure, $\hat{\mathscr{P}}$ is a pants decomposition of $\hat{F}$. Furthermore, we may assume that the components of $\partial F$ are horocycles in the corresponding cusps of $\hat{F}$. For each component $\alpha$ of $\mathscr{A}$, the two components of $A_{\alpha} \backslash \alpha$ lie in cusps of $\hat{F}$ (Figure 11). Hence, we may assume that the components of $\hat{\mathscr{A}}$ are horocycles as well.

For each component $\alpha$ of $\hat{\mathscr{P}}$ we take the given annular neighborhood on $F$ as an annular neighborhood on $\hat{F}$. In each cusp of $\hat{F}$ coming from a cusp of $F$ we choose the given punctured disc on $F$. In each cusp of $\hat{F}$ arising from a component $\alpha$ of $\mathscr{A}$, we take one of the components of $A_{\alpha} \backslash \alpha$. The closed pants of this paving of $\hat{F}$ are just the pants of the paving of $F$.

We may choose the local models for $\hat{F}$ to be the local models for $F$. The only difference occurs in the "new" cusps arising from components of $\mathscr{A}$. In these cusps, 
we choose the models suggested by Figure 11. By these associations, we may associate to each train track $T$ in $\mathscr{T}(\mathscr{P})$, a train track $\hat{T}$ on $\hat{F}$. Furthermore, since the edges of $\hat{T}$ are naturally identified with a subset of the edges of $T$, we may restrict any transverse measure $\nu$ on $T$ to a transverse measure $\hat{\nu}$ on $\hat{T}$. It is evident that this correspondence is continuous on cones and respects the identification of common subtracks. If $\mathscr{L}$ is a measured lamination $(\gamma, \mu)$ carried by $T$, then $\hat{\mathscr{L}}$ is a measured lamination $(\hat{\gamma}, \hat{\mu})$ carried by $\hat{T}$ with $\left(\hat{\mu}_{\#}\right)=(\hat{\mu})_{\#}$.

Let $U(\mathscr{A})$ be the collection of measured laminations whose supports are contained in $\mathscr{A}$. Let $\Delta(\mathscr{A})$ be the corresponding subset of $\mathscr{P} \mathscr{L}(F)$. From the above discussion, it is an easy exercise to verify the following lemma.

LEMMA 6.1. The following reduction map is continuous:

$$
\Lambda: \mathscr{P} \mathscr{L}(F) \backslash \Delta(\mathscr{A}) \rightarrow \mathscr{P} \mathscr{L}(\hat{F}) ; \quad \Lambda:[\mathscr{L}] \mapsto[\hat{\mathscr{L}}]
$$

Let $\mathscr{A}$ be a family of isotopy classes of disjoint, homotopically nontrivial simple closed curves on $F$. We shall represent any such family by geodesics in the compact region of $F$ and horocycles in the cusps of $F . \mathscr{M} \mathscr{L}(F ; \mathscr{A})$ will denote the subset of $\mathscr{M} \mathscr{L}(F)$ consisting of measured laminations whose supports are disjoint from $\mathscr{A}$. In particular, $\mathscr{M} \mathscr{L}(F ; \varnothing)=\mathscr{M} \mathscr{L}(F)$ and $\mathscr{M} \mathscr{L}(F ; \partial F)=\mathscr{M} \mathscr{L}_{0}(F)$, where $\mathscr{M} \mathscr{L}_{0}(F)$ is the set of measured laminations on $F$ which have compact supports. Briefly, a geodesic lamination is compact if no leaf of the lamination goes out to infinity in a cusp of $F$.

Note. For a closed surface $F$, all geodesic laminations are compact. Thus, in this case, $\mathscr{M} \mathscr{L}(F)=\mathscr{M} \mathscr{L}_{0}(F)$.

The action of $\mathbf{R}_{+}$clearly restricts to an action on $\mathscr{M} \mathscr{L}_{0}(F)$, and we obtain $\mathscr{P} \mathscr{L}_{0}(F)$ as the corresponding orbit space. In like manner we obtain $\mathscr{P} \mathscr{L}(F ; \mathscr{A})$ as the projective space corresponding to $\mathscr{M} \mathscr{L}(F ; \mathscr{A}) . \mathscr{M} \mathscr{L}(F ; \mathscr{A})$ has the subspace topology and $\mathscr{P} \mathscr{L}(F ; \mathscr{A})$ has the corresponding quotient topology. The reader will observe that $\mathscr{P} \mathscr{L}(F ; \mathscr{A})$ embeds in $\mathscr{P} \mathscr{L}(F)$.

Although the reduction maps are not injective, certain natural restrictions of them are.

LEMMA 6.2. The following restriction is a homeomorphism:

$$
\Lambda: \mathscr{P} \mathscr{L}(F ; \mathscr{A}) \rightarrow \mathscr{P} \mathscr{L}(\hat{F} ; \hat{\mathscr{A}}) ; \quad \Lambda:[\mathscr{L}] \mapsto[\hat{\mathscr{L}}] .
$$

COROLlaRY 6.3. The following restriction is a homeomorphism:

$$
\Lambda: \Lambda^{-1}\left(\mathscr{P} \mathscr{L}_{0}(\hat{F})\right) \rightarrow \mathscr{P} \mathscr{L}_{0}(\hat{F}) ; \quad \Lambda:[\mathscr{L}] \mapsto[\hat{\mathscr{L}}]
$$

7. The anatomy of a join. We are now ready to begin our discussion of the "anatomy" of a join. Again, we consider a fixed mapping class $\tau$ and the essential reduction system $\mathscr{A}_{\tau}$ for $\tau$. Now, consider the reduction of $\tau$ along $\mathscr{A}$, where $\mathscr{A}=\mathscr{A}_{\tau}$. For the purposes of the following discussion, we may, if necessary, replace $\tau$ by $\tau^{m}$, where $m$ is a positive integer. Therefore, by Theorem 2.1 , we may assume $\hat{\tau}$ preserves each of the connected components of $\hat{F}$, and each restriction of $\hat{\tau}$ is either trivial or pseudo-Anosov. 
Index the components of $\hat{F}$ as follows:

(i) $\hat{F}=\left(\amalg_{j \in J} \hat{F}_{j}\right) \amalg\left(\amalg_{k \in K} \hat{F}_{k}\right)$.

(ii) For each $j \in J, \hat{\tau}_{j}=1$.

(iii) For each $k \in K, \hat{\tau}_{k}$ is pseudo-Anosov.

Note. It is possible, of course, that $J=\varnothing$ or $K=\varnothing$.

By definition of pseudo-Anosov, for each $k$, as in (iii), there is a pair of nontrivial measured laminations $\left\{\mathscr{L}_{k+}, \dot{\mathscr{L}}_{k-}\right\} \subset \mathscr{M} \mathscr{L}\left(\hat{F}_{k}\right)$, and a positive real number $\lambda_{k}>1$, such that $\hat{\tau}_{k}\left(\mathscr{L}_{k+}\right)=\lambda_{k} \cdot \mathscr{L}_{k+}$ and $\hat{\tau}_{k}\left(\mathscr{L}_{k-}\right)=\lambda_{k}^{-1} \mathscr{L}_{k-}$.

Note. These measured laminations are compactly supported, $\left\{\mathscr{L}_{k+}, \mathscr{L}_{k-}\right\} \subset$ $\mathscr{M} \mathscr{L}_{0}\left(\hat{F}_{k}\right)[$ Th2, FLP].

We may extend the notion of the join of subsets of $\mathscr{P} \mathscr{L}(F)$, which we defined in $\S 4$, to arbitrary surfaces as follows. If $\mathscr{L}_{1}$ and $\mathscr{L}_{2}$ are measured laminations $\left(\gamma_{1}, \mu_{1}\right)$ and $\left(\gamma_{2}, \mu_{2}\right)$, such that $\gamma_{1} \cap \gamma_{2}=\varnothing$, we shall refer to $\mathscr{L}_{1}+\mathscr{L}_{2}$ as the direct sum of $\mathscr{L}_{1}$ and $\mathscr{L}_{2}, \mathscr{L}_{1} \oplus \mathscr{L}_{2}$, and write $\mathscr{L}_{1} \oplus \mathscr{L}_{2}=\left(\gamma_{1} \amalg \gamma_{2}, \mu_{1} \oplus \mu_{2}\right)$.

Two subsets $A$ and $B$ of $\mathscr{P} \mathscr{L}(F)$ have disjoint supports if $\operatorname{supp}\left(\mathscr{L}_{1}\right) \cap \operatorname{supp}\left(\mathscr{L}_{2}\right)=$ $\varnothing$ for each pair of measured laminations $\left(\mathscr{L}_{1}, \mathscr{L}_{2}\right)$ with $\left[\mathscr{L}_{1}\right] \in A$ and $\left[\mathscr{L}_{2}\right] \in B$. If $A$ and $B$ have disjoint support, then we may define the join of $A$ and $B, A * B$, by the rule

$$
A * B=\left\{[\mathscr{L}] \in \mathscr{P} \mathscr{L}(F) \mid \mathscr{L}=\mathscr{L}_{1} \oplus \mathscr{L}_{2},\left[\mathscr{L}_{1}\right] \in A,\left[\mathscr{L}_{2}\right] \in B\right\} .
$$

It is clear that we may employ this notion to define the following subsets of $\mathscr{P} \mathscr{L}(F)$.

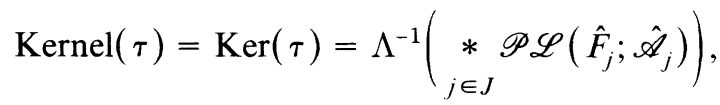

$$
\begin{aligned}
& \operatorname{Attractor}(\tau)=\operatorname{Att}(\tau)=\Lambda^{-1}\left(\underset{k \in K}{*}\left\{\left[\mathscr{L}_{k+}\right]\right\}\right) \text {, } \\
& \operatorname{Neutral}(\tau)=\operatorname{Neut}(\tau)=\underset{\alpha \in \mathscr{A}_{\tau}}{*}\{[\alpha]\}, \\
& \operatorname{Join}_{+}(\tau)=\operatorname{Ker}(\tau) * \operatorname{Att}(\tau) * \operatorname{Neut}(\tau) .
\end{aligned}
$$

The arguments of Lemma 1.3 establish that $A * B$ is, in fact, homeomorphic to the topological join of $A$ and $B$. In fact, using Lemma 6.2, we may easily establish the following result.

LeMma 7.1. (1) $\operatorname{Ker}(\tau) \cong *_{j \in J} \mathscr{P} \mathscr{L}\left(\hat{F}_{j} ; \hat{\mathscr{A}}_{j}\right)$.

(2) $\operatorname{Att}(\tau) \cong *_{k \in K}\left\{\left[\mathscr{L}_{k+}\right]\right\}^{j \in J}$.

(3) $\operatorname{Neut}(\tau) \cong{ }_{\alpha \in \mathscr{P}_{\tau}}\{[\boldsymbol{\alpha}]\}$.

(4) $\operatorname{Join}_{+}(\tau) \cong \operatorname{Ker}(\tau) * \operatorname{Att}(\tau) * \operatorname{Neut}(\tau)$.

REMARK. By Corollary 1.4, Lemma 7.1 and basic properties of topological joins we have the following observations.

(1) $\operatorname{Ker}(\tau)$ is homeomorphic to a sphere or a disc of some appropriate finite dimension (else it is empty).

(2) $\operatorname{Att}(\tau)$ is homeomorphic to a standard $n$-simplex, where $n+1$ is the number of pseudo-Anosov components in the reduction of $\tau$ along its essential reduction system (else empty). 
(3) $\operatorname{Neut}(\tau)$ is homeomorphic to a standard $n$-simplex, where $n+1$ is the number of connected components of the essential reduction system for $\tau$ (else empty).

(4) Join $+(\tau)$ is homeomorphic to a sphere or a disc of some appropriate finite dimension. In fact, if $\tau$ is infinite order, then either $\operatorname{Att}(\tau)$ or $\operatorname{Neut}(\tau)$ is nonempty. Hence, in this case, $\operatorname{Join}_{+}(\tau)$ is a disc. If $\tau$ is of finite order, then $\operatorname{Join}_{+}(\tau)$ is equal to $\mathscr{P} \mathscr{L}(F)$.

The Join _ of $\tau$ is defined by replacing $\operatorname{Att}(\tau)$ by the Repeller of $\tau$ which is defined as

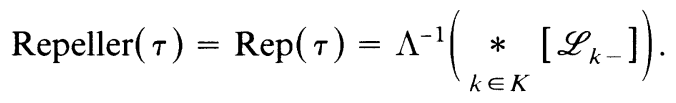

The following observations are easily checked.

Lemma 7.2. (1) For each $n>0$, Join ${ }_{ \pm}\left(\tau^{n}\right)=\operatorname{Join}_{ \pm}(\tau)$.

(2) For each $n<0$, Join ${ }_{ \pm}\left(\tau^{n}\right)=\operatorname{Join}_{\mp}(\tau)$.

(3) For each $\sigma \in \Gamma(F), \sigma\left(\operatorname{Join}_{ \pm}(\tau)\right)=\operatorname{Join}_{ \pm}\left(\sigma \tau \sigma^{-1}\right)$.

REMARK. The reader may easily check that the definitions we have given in this section extend the definitions given in $\S \S 3,4$ and 5 .

8. The Uniform Convergence Lemma. In this section, we shall prove the Uniform Convergence Lemma for an arbitrary element $\tau$ of $\Gamma(F)$.

Uniform Convergence Lemma. Let $\tau \in \Gamma(F)$, let $K$ be a compact subset of $\mathscr{P} \mathscr{L}(F) \backslash$ Join $_{-}(\tau)$ and let $U$ be an open neighborhood of $\operatorname{Join}_{+}(\tau)$. There exists a positive integer $N$, such that if $n \geqslant N$, then $\tau^{n}(K) \subset U$.

Proof. Let $\mathscr{A}$ and $\mathscr{B}$ denote the reduction systems for $\tau$ which are described in the introductory statements of $\S 6$. Let $\hat{\tau}$ be the reduction of $\tau$ along $\mathscr{B}$. As we observed in these introductory statements, $\hat{\tau}$ satisfies the Uniform Convergence Lemma. Let $\Lambda$ denote the corresponding reduction map

$$
\Lambda: \mathscr{P} \mathscr{L}(F) \backslash \Delta(\mathscr{B}) \rightarrow \mathscr{P} \mathscr{L}(F) .
$$

It is clear from the definition of the Joins that

$$
\Lambda^{-1}\left(\operatorname{Join}_{ \pm}(\hat{\tau})\right) \subset \operatorname{Join}_{ \pm}(\tau) .
$$

Since $\Delta(\mathscr{B}) \subset \operatorname{Join}_{+}(\tau)$ (in fact, $\Delta(\mathscr{B}) \subset \operatorname{Neut}(\tau)$ ), $\mathscr{P} \mathscr{L}(F) \backslash U$ is a compact subset of $\mathscr{P} \mathscr{L}(F) \backslash \Delta(\mathscr{B})$. Let $\hat{K}=\Lambda(K)$ and $C=\Lambda(\mathscr{P} \mathscr{L}(F) \backslash U)$. Observe (1) implies that $C \subset \mathscr{P} \mathscr{L}(\hat{F}) \backslash$ Join $_{+}(\hat{\tau})$. Furthermore, since $C$ is compact, $\mathscr{P} \mathscr{L}(\hat{F}) \backslash C$ is an open neighborhood of $\operatorname{Join}_{+}(\hat{\tau})$. Let $\hat{U}=\mathscr{P} \mathscr{L}(\hat{F}) \backslash C$.

As observed, there exists a positive integer $N$, such that for each integer $n \geqslant N$, $(\hat{\tau})^{n}(K) \subset \hat{U}$. Therefore

$$
\begin{gathered}
\tau^{n}(K) \subset \Lambda^{-1}\left(\Lambda\left(\tau^{n}(K)\right)\right)=\Lambda^{-1}\left(\hat{\tau}^{n}(\hat{K})\right), \\
\tau^{n}(K) \subset \Lambda^{-1}(\hat{U}) \subset U \text { for each integer } n \geqslant N .
\end{gathered}
$$

REMARK. The argument which has been employed here will not work for an arbitrary reduction system. For, in general, it is not true that $\Lambda^{-1}\left(\operatorname{Join}_{ \pm}(\hat{\tau})\right) \subset$ Join $_{ \pm}(\tau)$. For example, let $F$ be a closed, connected surface of genus two, and 

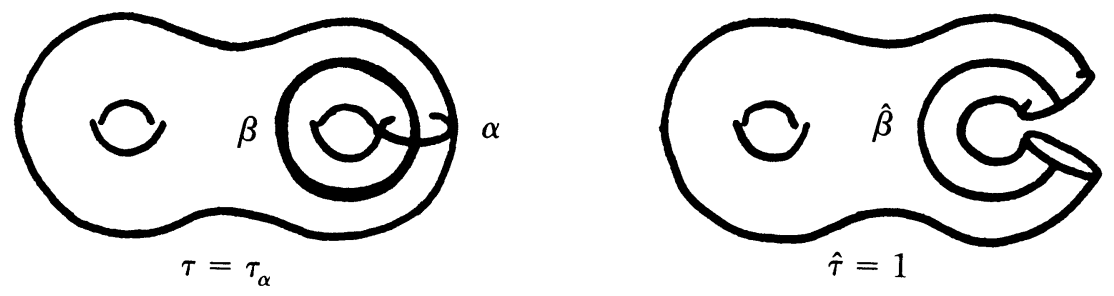

FIGURE 12

$\{\alpha, \beta\}$ be a pair of dual curves as in Figure 12. Let $\tau$ be the Dehn twist about $\alpha$, and $\Lambda$ be the reduction map corresponding to $\{\alpha\}$. The reduction of $\tau$ along $\alpha$ is trivial. Therefore

$$
\operatorname{Join}_{+}(\hat{\tau})=\mathscr{P} \mathscr{L}(F \backslash \alpha), \quad \Lambda^{-1}\left(\operatorname{Join}_{+}(\hat{\tau})\right)=\mathscr{P} \mathscr{L}(F) \backslash \Delta(\{\alpha\}) .
$$

On the other hand, $\beta \in \mathscr{P} \mathscr{L}(F) \backslash \Delta(\{\alpha\})$, but, since $\beta$ is dual to $\alpha, \beta \notin \operatorname{Join}_{+}(\tau)$.

9. Two generator subgroups of $\Gamma(F)$. In addition to the properties of Lemma 4.3, we shall need the following result concerning pseudo-Anosov mapping classes on connected surfaces.

LEMMA 9.1 [Mc1]. Let $F$ be a connected surface, $\tau$ a pseudo-Anosov mapping class, and $\left\{\left[\mathscr{L}_{+}\right],\left[\mathscr{L}_{-}\right]\right\}$the unique pair of fixed points for the action of $\tau$ on $\mathscr{P} \mathscr{L}(F)$. The stabilizer of $\left[\mathscr{L}_{ \pm}\right]$is a virtually cyclic subgroup of $\Gamma(F)$, any torsion free subgroup of which is cyclic.

We now proceed with the proof of Theorem B.

Proof of Theorem B. Let Join $(\sigma)=\operatorname{Join}_{-}(\sigma) \cup \operatorname{Join}_{+}(\sigma)$. First, we consider the case where $\operatorname{Join}(\sigma) \cap \operatorname{Join}(\tau)$ is empty. In particular, $\sigma$ and $\tau$ are of infinite order. Since $\operatorname{Join}(\sigma)$ and $\operatorname{Join}(\tau)$ are closed subsets of $\mathscr{P} \mathscr{L}(F)$, we may separate them by open subsets $U$ and $V$. That is:

(a) $\operatorname{Join}(\sigma) \subset U$.

(b) $\operatorname{Join}(\tau) \subset V$.

(c) $U \cap V=\varnothing$.

Since $\mathscr{P} \mathscr{L}(F)$ is connected, we may choose

(d) $p \in \mathscr{P} \mathscr{L}(F) \backslash(U \cup V)$.

Note. (e) $\bar{V} \cup\{p\}$ is a compact subset of $\mathscr{P} \mathscr{L}(F) \backslash$ Join $_{ \pm}(\sigma)$.

(f) $\bar{U} \cup\{p\}$ is a compact subset of $\mathscr{P} \mathscr{L}(F) \backslash \operatorname{Join}_{ \pm}(\tau)$.

By repeated applications of the Uniform Convergence Lemma, we conclude that there exists:

(g) $M_{1}>0$ such that $\sigma^{n}(\bar{V} \cup\{p\}) \subset U$ for all $n \geqslant M_{1}$.

(h) $M_{2}>0$ such that $\sigma^{-n}(\bar{V} \cup\{p\}) \subset U$ for all $n \geqslant M_{2}$.

(i) $N_{1}>0$ such that $\tau^{n}(\bar{U} \cup\{p\}) \subset V$ for all $n \geqslant N_{1}$.

(j) $N_{2}>0$ such that $\tau^{-n}(\bar{U} \cup\{p\}) \subset V$ for all $n \geqslant N_{2}$.

Let $M=M_{1} M_{2}$ and $N=N_{1} N_{2}$. Then:

(k) For each nonzero integer $n,\left(\sigma^{M}\right)^{n}(V \cup\{p\}) \subset U$.

(l) For each nonzero integer $n,\left(\tau^{N}\right)^{n}(U \cup\{p\}) \subset V$. 
By the Freedom Criterion, $\operatorname{gp}\left(\sigma^{M}, \tau^{N}\right)$ is a free group of rank two on the generators $\sigma^{M}$ and $\tau^{N}$.

Now, we consider an arbitrary pair $\sigma$ and $\tau$ in $\Gamma(F)$. We shall prove Theorem B by induction on $C(F)$, where $C(F)$ is the maximum number of components of an admissible system on $F$. One may compute that $C(F)=3 g+b-3 c$. If $\mathscr{A}$ is an admissible system on $F$, then $C(\hat{F})<C(F)$. Therefore, $C(F)$ is ideally suited for "reduction" arguments.

Suppose that $C(F)=0$. Then $F$ is a disjoint union of pairs of pants. Since the mapping class group of a pair of pants is finite [BLM], it follows easily that $\Gamma(F)$ is finite. $(\Gamma(F)$ is an extension of the direct product of the mapping class groups of the components of $F$ by a subgroup of the group of permutations of the components.) Therefore, Theorem B follows by triviality.

Now, suppose that $C(F)>0$. In addition, suppose that $F$ is not connected. Since hypothesis and conclusion are invariant under exponentiation of $\sigma$ and $\tau$ by nonzero integers, we can assume that $\sigma$ and $\tau$ preserve each of the components of $F$. We have natural projections $\pi_{1}: \operatorname{gp}(\sigma, \tau) \rightarrow \Gamma\left(F_{i}\right)$. Restrict these to epimorphisms $\pi_{i}: \operatorname{gp}(\sigma, \tau)$ $\rightarrow \operatorname{gp}\left(\sigma_{i}, \tau_{i}\right)$.

Suppose that we can choose a pair of nonzero integers $M_{i}$ and $N_{i}$ for each $i \in I$, such that $\operatorname{gp}\left(\sigma_{i}^{M_{i}}, \tau_{i}^{N_{i}}\right)$ is abelian. Then $\operatorname{gp}\left(\sigma^{M}, \tau^{N}\right)$ is abelian, where $M=\prod_{i \in I} M_{i}$ and $N=\prod_{i \in I} N_{i}$.

On the other hand, if for some index $i$ there exists a pair of nonzero integers $M_{i}$ and $N_{i}$, such that the corresponding group $\operatorname{gp}\left(\sigma_{i}^{M_{i}}, \tau_{i}^{N_{i}}\right)$ is a free group of rank 2 , then the epimorphism $\pi_{i}$ splits. Therefore, the natural $\operatorname{splitting} \operatorname{gp}\left(\sigma^{M_{i}}, \tau^{N_{i}}\right)$ is a free group of rank 2 .

In other words, we may assume that $F$ is connected. Furthermore, by the previous argument, we may assume that $\operatorname{Join}(\sigma) \cap \operatorname{Join}(\tau)$ is nonempty. Furthermore, we may assume that $\sigma$ and $\tau$ are of infinite order. (Otherwise, the conclusion is trivial.)

Suppose that $\sigma$ is pseudo-Anosov, and $\operatorname{Join}(\sigma) \cap \operatorname{Join}(\tau)$ is nonempty. If $[\mathscr{L}] \in$ $\operatorname{Join}(\sigma) \cap \operatorname{Join}(\tau)$, then clearly $\mathscr{L}$ is one of the pair of measured laminations associated to $\sigma$. It is clear from the descriptions of the Joins that $\tau$ is pseudo-Anosov, with $[\mathscr{L}]$ as one of its pair of fixed points. Therefore, $\operatorname{gp}(\sigma, \tau)$ is contained in the stabilizer of the projective class of $\mathscr{L}$. By Lemma 9.1, we conclude that $\operatorname{gp}(\sigma, \tau)$ is virtually cyclic. By passing to exponents if necessary, we conclude that $\operatorname{gp}\left(\sigma^{m}, \tau^{n}\right)$ is cyclic.

Therefore, we may assume that $\sigma$ and $\tau$ are infinite order reducible mapping classes. In particular, $\mathscr{A}_{\sigma} \neq \varnothing$. Suppose that $[\mathscr{L}] \in \operatorname{Join}(\sigma) \cap \operatorname{Join}(\tau)$. From the description of the Joins and the action of $\Gamma(F)$ on measured laminations, we can choose representatives $s \in \sigma$ and $t \in \tau$, and positive integers $m$ and $n$, such that $s^{m}(\gamma)=\gamma=t^{n}(\gamma)$, where $\gamma$ is the support of $\mathscr{L}$. Let $\mathscr{A}$ be the collection of compact leaves of $\gamma$. Clearly, $\mathscr{A}$ is preserved by $s^{m}$ and $t^{n}$. Therefore, if $\mathscr{A}$ is nonempty, $\sigma^{m}$ and $\tau^{n}$ have a common nontrivial reduction system. On the other hand, if $\gamma$ does not contain compact leaves, then there are components of $F \backslash \gamma$ which contain simple closed geodesics $\left(\mathscr{A}_{\sigma} \subset F \backslash \gamma\right)$. To each such component $N$, we may associate a closed retract $M$ which is unique up to isotopy fixing the frontier of $N$, and is a 


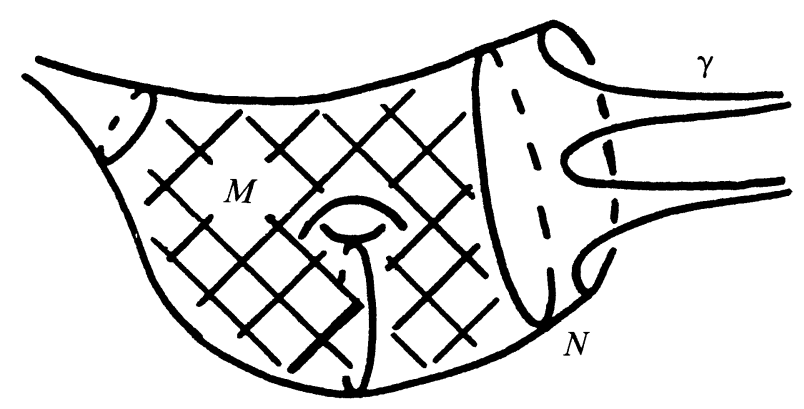

Figure 13

manifold with boundary $\partial M$ (Figure 13). If every boundary component of $M$ bounded a disk of $F$ or a cusp of $F$, then $\gamma$ would be contained in $M$. (Cusps connected.) Any boundary component of $M$ which does not bound a disk of $F$ or a cusp of $F$ is isotopic to a unique simple closed geodesic. Hence, the collection of such boundary components corresponds to a common nontrivial reduction system for $\sigma^{m}$ and $\tau^{n}$. In any event, $\sigma^{m}$ and $\tau^{n}$ have a common nontrivial reduction system $\mathscr{A}$.

REMARK. It may occur that, in the latter event of the above discussion, certain of the boundary components of the various components are parallel on $F$. Thus, the correspondence of this collection of boundary components with a reduction system need not be one-to-one.

Let $\Gamma_{\mathscr{A}}(F)$ be the stabilizer of $\mathscr{A}$ in $\Gamma(F)$ and consider the associated reduction homomorphism [BLM]

$$
\Lambda: \Gamma_{\mathscr{A}}(F) \rightarrow \Gamma(\hat{F}), \quad[t] \mapsto[\hat{t}] .
$$

Again, restrict this homomorphism to an epimorphism

$$
\Lambda: \operatorname{gp}(\sigma, \tau) \rightarrow \operatorname{gp}(\hat{\sigma}, \hat{\tau}) .
$$

Since $C(\hat{F})<C(F)$, the induction hypothesis implies that there exists a pair of nonzero integers $m$ and $n$, such that either $\operatorname{gp}\left(\hat{\sigma}^{m}, \hat{\tau}^{n}\right)$ is abelian or a free group of rank 2. In the latter case, the splitting argument just employed implies that $\operatorname{gp}\left(\sigma^{m}, \tau^{n}\right)$ is a free group of rank 2. In the former case, since the kernel of the reduction homomorphism is abelian [BLM], we conclude that $\operatorname{gp}\left(\sigma^{m}, \tau^{n}\right)$ is solvable. The Theorem of [BLM] stated in the introduction implies that $\operatorname{gp}\left(\sigma^{m}, \tau^{n}\right)$ is virtually abelian. By passing to higher exponents $M$ and $N$, if necessary, we conclude that $\operatorname{gp}\left(\sigma^{M}, \tau^{N}\right)$ is abelian.

10. A Tits-Alternative for $\Gamma(F)$.

Proof of Theorem A. As in the previous proof, we shall use induction on $C(F)$. Again, the case where $C(F)=0$ is trivial. Hence, we assume that $C(F)>0$. Since $\Gamma(F)$ is virtually torsion free [BL, BLM], we may assume that $G$ is torsion free. In addition, we shall assume that $G$ does not contain a nonabelian free group. Finally, by the same argument employed in the proof of Theorem B, we may assume that $F$ is connected. 
First, suppose that $G$ contains a pseudo-Anosov $\sigma$. If $\tau \in G$, by Theorem B, we may choose a pair of nonzero integers $M$ and $N$, such that $\operatorname{gp}\left(\sigma^{M}, \tau^{N}\right)$ is abelian. Since $\mathrm{Join}_{+}(\sigma)=\{[\mathscr{L}]\}$, Lemma 7.2 implies that $\tau^{N}([\mathscr{L}])=[\mathscr{L}]$. If $\tau$ is nontrivial, then by Lemma 9.1 and the assumption that $G$ is torsion free, $\tau^{N}$ is pseudo-Anosov. This implies that $\tau$ is pseudo-Anosov and $[\mathscr{L}]$ is one of its fixed points. Since $\tau$ is an arbitrary nontrivial element of $G$, we conclude that $G$ is contained in the stabilizer of $[\mathscr{L}]$ in $\Gamma(\mathscr{L})$. Therefore, by Lemma $9.1, G$ is virtually cyclic.

Therefore, we may assume that $G$ does not contain a pseudo-Anosov. Together with our previous assumptions, we see that $G$ consists entirely of infinite order, reducible mapping classes. In particular, if $\sigma \in G$, then the essential reduction system $\mathscr{A}_{\sigma}$ for $\sigma$ is nonempty (Theorem 2.1 ).

Suppose that $\sigma$ and $\tau$ are an arbitrary pair of elements of $G$. By Theorem B, choose a pair of nonzero integers $M$ and $N$, such that $\operatorname{gp}\left(\sigma^{M}, \tau^{N}\right)$ is abelian. By the properties of essential reduction systems $\left[\right.$ BLM] $, \mathscr{A}_{\sigma}=\mathscr{A}_{\sigma^{M}}, \mathscr{A}_{\tau}=\mathscr{A}_{\tau^{N}}$ and $\sigma^{M}\left(\mathscr{A}_{\tau}\right)$ $=\mathscr{A}_{\tau}$. Likewise, we conclude that $\left\langle\mathscr{A}_{\sigma}, \mathscr{A}_{\tau}\right\rangle=0$.

Let $\mathscr{A}_{G}=\cup_{\sigma \in G} \mathscr{A}_{\sigma}$. By the previous remarks, $\mathscr{A}_{G} \neq \varnothing, \mathscr{A}_{G}$ is an admissible system. Finally, by the same remarks, $\mathscr{A}_{G}$ is stabilized by each element $\tau$ of $G$. Let $\mathscr{A}=\mathscr{A}_{G}$, and consider the associated reduction homomorphism $\Lambda: \Gamma_{\mathscr{A}}(F) \rightarrow \Gamma(\hat{F})$, and its corresponding epimorphic restriction $\Lambda: G \rightarrow \hat{G}$.

Since $C(\hat{F})<C(F)$, the induction hypothesis implies that either $\hat{G}$ is virtually abelian or $\hat{G}$ contains a nonabelian free group. In the latter case, we may employ a splitting argument, as in the proof of Theorem B, to prove that $G$ contains a nonabelian free group. In the former case, since $\operatorname{ker}(\Lambda)$ is abelian, $G$ is virtually solvable. As in the previous proof, the Theorem of [BLM] implies that $G$ is virtually abelian.

ACKNOWLEDGEMENTS. This work is part of the author's doctoral dissertation at Columbia University [Mc2]. We thank Joan Birman, the author's thesis advisor, for her continual encouragement and Alex Lubotzky for suggesting the problem of a “Tits-Alternative" for surface mapping class groups. We owe a great deal to John Harer and Robert Penner whose theory of pavings provided the framework for many of the techniques used in this paper.

The contents of this paper were presented on November 12, 1982 at the 799th Meeting of the American Mathematical Society, Michigan State University, East Lansing. The lecture, "Free Subgroups of Surface Mapping Class Groups" was delivered to the special session on geometric topology and was sponsored by the author's thesis advisor, Joan Birman.

The author gratefully acknowledges the support of the Massachusetts Institute of Technology and the Max-Planck-Institut für Mathematik, Bonn, during the preparation of this paper.

\section{REFERENCES}

[BL] H. Bass and A. Lubotzky, Automorphisms of groups and of schemes of finite type, Israel J. Math. 44 (1983), 1-22.

[B1] J. S. Birman, Braids, links and mapping class groups, Ann. of Math. Studies, No. 82, Princeton Univ. Press, Princeton, N.J., 1974.

[B2] __ The algebraic structure of surface mapping class groups, Discrete Groups and Automorphic Functions (W. Harvey, ed.), Academic Press, New York, 1977. 
[BLM] J. S. Birman, A. Lubotzky and J. McCarthy, Abelian and solvable subgroups of the mapping class group, Duke Math. J. 50 (1983), 1107-1120.

[FLP] A. Fathi, F. Laudenbach, V. Poenaru et al., Travaux de Thurston sur les surfaces, Astérisque 66-67, Société Mathématique de France, 1979.

[G] J. Gilman, On the Neilsen type and the classification for the mapping class group, Adv. in Math. 40 (1981), 68-96.

[H] J. Harer, Stability of the homology of the mapping class groups of orientable surfaces, Ann. of Math. 121 (1985), 215-249.

[HP] J. Harer and R. Penner, Combinatorics of train tracks and pavings of projective lamination spaces (to appear).

[Ha] W. J. Harvey, Geometric structures of surface mapping class groups, Homological Group Theory (C. T. Wall, ed.), London Math. Soc. Lecture Notes, No. 36, Cambridge Univ. Press, London, 1979, pp. 255-269.

[HT] A. Hatcher and W. Thurston, A presentation for the mapping class group of a closed orientable surface, Topology 19 (1980), 221-237.

[Ke] S. P. Kerckhoff, The Neilsen realization problem, Ann. of Math. (2) 117 (1983), 235-265.

[Mc1] J. McCarthy, Normalizers and centralizers of pseudo-Anosov mapping classes, preprint available upon request.

[Mc2] _ Subgroups of surface mapping class groups, Ph.D. thesis, Columbia University, May, 1983.

[M] J. Morgan, Train tracks and geodesic laminations, Columbia University Lecture Notes (to appear).

[N] J. Nielsen, Surface transformation classes of algebraically finite type, Danske Vid. Selsk. Math.-Phys. (2) 21 (1944), 1-89.

[P] R. Penner, A computation of the action of the mapping class group on isotopy classes of curves and arcs in surfaces, Ph.D. thesis, Massachusetts Institute of Technology, 1982.

[Th1] W. P. Thurston, The geometry and topology of 3-manifolds, Princeton Univ. Lecture Notes (to appear).

[Th2] __ On the geometry and dynamics of diffeomorphisms of surfaces, preprint.

[Th3] _ Three dimensional manifolds, Kleinian groups and hyperbolic geometry, Bull. Amer. Math. Soc. (N.S.) 6 (1982), 357-381.

[Th4] _ L Lectures Notes, Boulder, Colorado, 1980.

[Th5] __ Hyperbolic structures on 3-manifolds. II, preprint, July, 1980.

[T] J. Tits, Free subgroups in linear groups, J. Algebra 20 (1972), 250-270.

Max - Planck - Institut fUr Mathematik, Gottfried - Claren - Strasse 26, D - 5300 Bonn 3, GERMLANY

SONDERFORSCHUNGSBereich TheORETISChe MATHEMATIK, Beringstrasse 4, D-5300 Bonn 1, GERMANY

Current address: Department of Mathematics, Michigan State University, East Lansing, Michigan 48824 